\title{
Disease-linked TDP-43 hyperphosphorylation suppresses TDP-43 condensation and aggregation
}

\section{Authors:}

Lara Gruijs da Silva ${ }^{1,2}$, Francesca Simonetti ${ }^{1,3}$, Saskia Hutten ${ }^{1}$, Henrick Riemenschneider ${ }^{3}$, Erin L. Sternburg ${ }^{1}$, Lisa M. Pietrek ${ }^{4}$, Jakob Gebel ${ }^{5}$, Volker Dötsch ${ }^{5}$, Dieter Edbauer ${ }^{2,3,6}$, Gerhard Hummer ${ }^{4,7}$, Lukas S. Stelzl ${ }^{1,4,8,9}$, Dorothee Dormann ${ }^{1,6,9^{*}}$

\author{
Affiliations: \\ ${ }^{1}$ Johannes Gutenberg-Universität (JGU), Biocenter, Institute of Molecular Physiology, 55128 \\ Mainz, Germany \\ ${ }^{2}$ Graduate School of Systemic Neurosciences (GSN), 82152 Planegg-Martinsried, Germany \\ ${ }^{3}$ German Center for Neurodegenerative Diseases (DZNE), 81377 Munich, Germany \\ ${ }^{4}$ Max Planck Institute of Biophysics, Department of Theoretical Biophysics, 60438 Frankfurt \\ am Main, Germany \\ ${ }^{5}$ Institute for Biophysical Chemistry, Goethe-Universität, 60438 Frankfurt am Main, \\ Germany \\ ${ }^{6}$ Munich Cluster for Systems Neurology (SyNergy) Munich, 81377 Munich, Germany \\ ${ }^{7}$ Institute for Biophysics, Goethe-Universität, 60438 Frankfurt am Main, Germany \\ ${ }^{8}$ Johannes Gutenberg-Universität (JGU), KOMET1, Institute of Physics, 55128 Mainz, \\ Germany \\ ${ }^{9}$ Institute of Molecular Biology (IMB), 55128 Mainz, Germany
}

*Correspondence to: ddormann@uni-mainz.de 


\begin{abstract}
Post-translational modifications (PTMs) have emerged as key modulators of protein phase separation and have been linked to protein aggregation in neurodegenerative disorders. The major aggregating protein in amyotrophic lateral sclerosis (ALS) and frontotemporal dementia (FTD), the RNA-binding protein TDP-43, is hyperphosphorylated in disease on several Cterminal serine residues, which is generally believed to promote TDP-43 aggregation. Here, we show that hyperphosphorylation by Casein kinase $1 \delta$ or C-terminal phosphomimetic mutations surprisingly reduce TDP-43 phase separation and aggregation and render TDP-43 condensates more liquid-like and dynamic. Multi-scale simulations reveal reduced homotypic interactions of TDP-43 low complexity domains through enhanced solvation of phosphomimetic residues. Cellular experiments show that phosphomimetic substitutions do not affect nuclear import or RNA regulatory functions of TDP-43, but suppress accumulation of TDP-43 in membrane-less organelles and promote its solubility in neurons. We propose that TDP-43 hyperphosphorylation may be a protective cellular response to counteract TDP-43 aggregation.
\end{abstract}

\title{
Keywords
}

Neurodegeneration / phase separation / phosphorylation / RNA-binding protein / TDP-43 


\section{Introduction}

TAR DNA binding protein (TDP-43) is the major aggregating protein in ALS and FTD patients and also forms pathological aggregates in up to 50\% of Alzheimer's disease patients (Josephs et al., 2014; Neumann et al., 2006). It is a ubiquitously expressed RNA-binding protein (RBP) with key functions in RNA processing, e.g. regulation of alternative splicing and polyadenylation, miRNA processing, mRNA stability and localization (Ratti and Buratti, 2016). In the affected brain regions of ALS and FTD patients, the physiological diffuse nuclear localization of TDP-43 is lost. Instead the protein forms cytoplasmic and occasionally nuclear inclusions in neurons and glial cells (Mackenzie et al., 2010). TDP-43 pathology closely correlates with neurodegeneration, and both loss-of-function mechanisms, e.g. misregulation of nuclear RNA targets, and gain-of-function mechanisms, e.g. aberrant interactions of the TDP43 aggregates, are believed to contribute to neuronal dysfunction and eventually neurodegeneration (Ling et al., 2013; Tziortzouda et al., 2021).

Like other prion-like RBPs, TDP-43 is thought to aggregate through aberrant liquid-liquid phase separation (LLPS), i.e. the transition of liquid-like RBP condensates into a solid-like state (Nedelsky and Taylor, 2019). Aberrant phase transitions may occur in stress granules (SGs) or other membrane-less organelles (MLOs), where aggregation-prone RBPs are highly concentrated and exceed the critical concentration for LLPS (Alberti and Dormann, 2019; Alberti and Hyman, 2021). Subsequent liquid-to-solid phase transition, as demonstrated for various disease-linked RBPs in vitro (Molliex et al., 2015; Patel et al., 2015), may then cause formation of pathological RBP inclusions. LLPS is often driven by intrinsically disordered low complexity domains (LCDs), that tend to engage in weak multivalent interactions with other molecules (Alberti, 2017). TDP-43 harbors a long C-terminal LCD enriched in glycine, serine, asparagine and glutamine residues, which drives intermolecular TDP-43 interactions and assembly by phase separation (Babinchak et al., 2019; Conicella et al., 2016). The LCD is also the region that harbors numerous ALS-linked point mutations (Buratti, 2015), suggesting that small chemical changes to the TDP-43 LCD can cause neurodegeneration.

LLPS and MLO dynamics are often regulated by post-translational modifications (PTMs) in LCDs, as the introduction of small chemical groups or proteins changes the chemical nature of amino acids, e.g. their charge or hydrophobicity, which can alter their molecular interactions and LLPS behavior (Bah and Forman-Kay, 2016; Hofweber and Dormann, 2019). A highly disease-specific PTM on deposited TDP-43 inclusions is hyperphosphorylation on C-terminal serine residues in the LCD (Hasegawa et al., 2008; Inukai et al., 2008; Kametani et al., 2016; Neumann et al., 2009). Antibodies specific for C-terminal TDP-43 phosphorylation sites (e.g. S409/S410 and S403/S404) detect inclusion pathology in patients, without cross-reactivity with physiological nuclear TDP-43. Therefore, C-terminal TDP-43 hyperphosphorylation is considered a pathological hallmark and is generally believed to promote TDP-43 aggregation (Buratti, 2018). This view is largely based on the observations that C-terminal TDP-43 phosphorylation correlates with inclusion pathology and that overexpression of kinases that can phosphorylate TDP-43 enhance TDP-43 aggregation and neurotoxicity (Choksi et al., 2014; Liachko et al., 2014; Nonaka et al., 2016; Taylor et al., 2018). Based on these studies, inhibition of TDP-43 phosphorylation by specific kinase inhibitors has even been proposed as a potential therapeutic strategy for ALS (Liachko et al., 2013; Martinez-Gonzalez et al., 2020; Salado et 
al., 2014). However, the molecular consequences of this disease-linked PTM are still poorly understood, and its effects on TDP-43 LLPS and aggregation are still unknown.

Using in vitro, in silico and cellular experiments, we now demonstrate that disease-linked C-terminal hyperphosphorylation of TDP-43 suppresses TDP-43 condensation and insolubility. We show this through a) in vitro phase separation and aggregation assays with recombinant, full-length TDP-43; b) coarse-grained and atomistic molecular dynamics simulations of condensates of TDP-43 LCDs, elucidating molecular driving forces; and c) experiments in $\mathrm{HeLa}$ cells and primary rat neurons, where C-terminal phosphomimetic mutations do not disturb nuclear import or RNA processing functions of TDP-43, but abrogate TDP-43 condensation into stress-induced membrane-less organelles and enhance its solubility. Based on our findings, we suggest that C-terminal TDP-43 hyperphosphorylation may be a protective cellular response to counteract TDP-43 solidification, rather than being a driver of TDP-43 pathology, as has so far been assumed. 


\section{Results}

\section{In vitro phosphorylation with Casein kinase $1 \delta$ reduces condensation of TDP-43}

To examine how phosphorylation affects TDP-43 phase transitions, we expressed and purified unphosphorylated full-length TDP-43 with a solubilizing MBP tag and a His6-tag in E. coli (Wang et al., 2018) (Fig. S1A-E). We then in vitro phosphorylated the purified protein with casein kinase 1 delta (CK1 1 ), a kinase previously reported to phosphorylate TDP-43 at diseaseassociated sites (Kametani et al., 2009), and confirmed phosphorylation of C-terminal serines (S403/S404; S409/S410) with phospho-specific antibodies (Fig. S2A). Mass spectrometric analysis detected phosphorylation on several additional serine/threonine sites (Fig. S2B), and the running behavior in SDS-PAGE suggests hyperphosphorylation on multiple sites (Fig. S2A, Fig. 1B). We then induced phase separation of the unphosphorylated vs. in vitro phosphorylated TDP-43 by cleaving off the MBP tag with TEV protease (Wang et al., 2018) and used centrifugation to separate the condensates (C) from the cleared supernatant (S) (Fig. 1A). Cleaved TDP-43 was mostly in the condensate fraction $(\mathrm{S} / \mathrm{C}$ ratio $<0.5)$, whereas in vitro phosphorylated TDP-43 was predominantly in the supernatant ( $\mathrm{S} / \mathrm{C}$ ratio $\sim 1.8$ ) (Fig. 1B, C). Reduced sedimentation of TDP-43 was not seen upon addition of ATP or CK1 $\delta$ alone, suggesting that it is indeed caused by the addition of phospho-groups to TDP-43.

\section{C-terminal phosphomimetic substitutions mimicking disease-linked phosphorylation suppress TDP-43 phase separation}

To study defined disease-linked phosphorylation sites, we generated phosphomimetic proteins harboring different numbers of phosphomimetic serine-to-aspartate (S-to-D) mutations (or corresponding serine-to-alanine (S-to-A) mutations as control) in the C-terminal region of TDP43. Phosphorylation on S409/S410 is a highly specific and consistent feature of aggregated TDP-43 in all ALS/FTD subtypes (Inukai et al., 2008; Neumann et al., 2009), and five phosphorylation sites (S379, S403, S404, S409 and S410) were detected with phosphorylation site-specific antibodies in human post-mortem tissue (Hasegawa et al., 2008). Therefore, we mutated these serines to create "2D" and "5D" variants as well as the corresponding "2A" and "5A" controls (Fig. 1D). Based on a mass spectrometric study that found phosphorylation on 12 out of 14 serines in the C-terminal LCD of TDP-43 in ALS spinal cord (Kametani et al., 2016), we also mutated these 12 sites (S373, S375, S379, S387, S389, S393, S395, S403, S404, S407, S409, S410) to create "12D" or "12A" variants (Fig. 1D). Interestingly, the PLAAC web tool (http://plaac.wi.mit.edu/) that allows prediction of probable prion subsequences using a hidden-Markov model (HMM) algorithm (Lancaster et al., 2014), predicted a reduced prionlike character of the phosphomimetic $12 \mathrm{D}$ variant in comparison to the wild-type (Wt) and 12A protein (Fig. S3).

To study phase separation experimentally, all variants were expressed and purified as TDP43-MBP-His6 fusion proteins (Fig. S1A-E), and phase separation induced by TEV proteasemediated cleavage of the MBP tag was examined by turbidity, sedimentation or microscopic condensate assays. Turbidity measurements revealed a concentration-dependent increase in phase separation for TDP-43 Wt, as expected, whereas the increase was less pronounced for the $2 \mathrm{D}$ and $5 \mathrm{D}$ variants and no concentration-dependent increase was seen for the $12 \mathrm{D}$ mutant (Fig. 1E). The gradual decrease in turbidity caused by the phosphomimetic mutations 
$(\mathrm{Wt}>2 \mathrm{D}>5 \mathrm{D}>12 \mathrm{D})$ was not seen to the same extent for the corresponding S-to-A control mutations (Fig. 1E), hence suppression of phase separation is not due to the loss of serines at these positions, but can be attributed to the additional negative charges introduced by the $\mathrm{D}$ substitutions. Turbidity assays in phosphate buffer instead of Hepes buffer gave similar results (Fig. S4A), and sedimentation assays confirmed that TDP-43 condensation is gradually suppressed by increasing numbers of phosphomimetic mutations (Fig. S4B, C).

\section{Phosphomimetic S-to-D substitutions lead to rounder TDP-43 condensates, whereas S-to- A mutations cause an amorphous, aggregate-like morphology}

Interestingly, bright field microscopy revealed that TDP-43 Wt formed relatively small, amorphous condensates, suggestive of solid-like material properties (Fig. 1F). In contrast, the phosphomimetic S-to-D proteins formed fewer, but much larger and rounder condensates (Fig. $1 F$, see G-I for quantification), suggesting a more liquid-like behavior and therefore fusion of condensates into larger droplets. Again, the observed changes were correlated with the number of phosphomimetic mutations, i.e. they were most pronounced for the $12 \mathrm{D}$ mutant, which formed very few, but large and perfectly circular protein droplets. (Note that these few large condensates most likely escape detection in the turbidity assay due to rapid sedimentation during the assay). In contrast, the S-to-A control variants formed numerous small, amorphous condensates and had a more irregular, aggregate-like appearance than TDP-43 Wt (Fig. 1F). This phenotype suggests that the $\mathrm{OH}$ groups in the respective serines influence the material properties of TDP-43 and contribute to preventing its aggregation. Similar results were obtained when the assay was carried out in phosphate buffer instead of Hepes buffer, except that 12D was unable to form any visible condensates in phosphate buffer (Fig. S4D). Together, these results demonstrate that phosphomimetic substitutions mimicking disease-linked C-terminal TDP-43 phosphorylation reduce the tendency of TDP-43 to phase separate into amorphous condensates and suggest a more dynamic, liquid-like behavior of C-terminally phosphorylated TDP-43.

\section{C-terminal phosphomimetic substitutions yield more liquid-like, dynamic TDP-43 condensates}

To test whether the phosphomimetic mutations indeed render TDP-43 more liquid-like, we performed live imaging of Alexa488-labelled Wt, 5D and 12D condensates by spinning disc confocal microcopy. For TDP-43 Wt, no fusion events were observed over a time course of several minutes. Instead the small condensates stuck to each other like "velcro balls" (movie 1, Fig. 2A). In contrast, 5D condensates occasionally and slowly fused with each other, and 12D condensates readily fused upon contact and relaxed into perfectly round spheres, indicating a liquid droplet-like nature (movies 2 and 3, Fig. 2A). To assess the mobility of TDP-43 molecules in condensates, we performed half-bleaches of condensates and analyzed fluorescent recovery after photobleaching (FRAP) in the bleached half. In TDP-43 Wt condensates, fluorescence recovered very slowly, indicating a low mobility of TDP-43 molecules, whereas recovery was faster in 5D and even faster in 12D condensates (Fig. 2B, C), in line with an increased mobility of phosphomimetic TDP-43 compared to "unmodified" TDP-43. Taken together, phosphomimetic S-to-D substitutions in the C-terminal region enhance the liquidity of TDP-43 condensates, suggesting that phosphorylation in this region might counteract TDP43 's tendency to form solid, irreversible aggregates. 


\section{C-terminal phosphomimetic substitutions reduce TDP-43 aggregation}

To address whether phosphorylation indeed counteracts TDP-43 aggregation, we performed in vitro aggregation assays modified from published protocols (French et al., 2019; Halfmann and Lindquist, 2008). Under the assay conditions, TEV cleavage of fluorescently labelled TDP-43MBP-His6 yields amorphous TDP-43 aggregates that can be visualized by confocal microscopy. In contrast to $\mathrm{Wt}$ or $12 \mathrm{~A}$, the phosphomimetic $5 \mathrm{D}$ or $12 \mathrm{D}$ proteins formed much smaller and fewer aggregates, respectively (Fig. 2D), suggesting that C-terminal TDP-43 phosphorylation can efficiently suppress TDP-43 aggregation. For biochemical characterization of the formed aggregates, we performed semi-denaturing detergent-agarose gel electrophoresis (SDD-AGE) under the same assay conditions, just in the absence of TEV, as MBP-tagged TDP43 aggregates slower than TDP-43 and distinct oligomeric/polymeric species resistant to $0.5 \%$ SDS can be visualized under these conditions (Fig. S5A). In comparison to TDP-43 Wt and 5D, 12D showed reduced and delayed oligomerization and formation of high molecular weight species (Fig. 2E, equal protein input shown in Fig. 2F). In contrast, 12A formed SDS-resistant oligomers/high molecular weight species at a higher rate, which together with our microscopic images of TDP-43 condensates (Fig. 1F), suggests that C-terminal alanine substitutions make TDP-43 more aggregation-prone. Taken together, C-terminal phosphomimetic substitutions that mimic the phosphorylation pattern in ALS patients reduce the formation of SDS-resistant high molecular weight oligomers and TDP-43 aggregates in vitro.

\section{Multiscale simulations of the TDP-43 low complexity domain reveal reduced protein- protein interactions through enhanced solvation of phosphomimetic residues}

To understand the effect of C-terminal TDP-43 phosphorylation on phase separation at the molecular level, we used coarse-grained and atomistic simulations of the disordered TDP-43 LCD (aa. 261 - 414) with and without phosphomimetic substitutions. We found that phosphomimetic substitutions locally reduce protein-protein interactions (Fig. S6) and increase protein-solvent interactions (Fig. 3). In the coarse-grained simulations, the LCD of both TDP$43 \mathrm{Wt}$ and 12D phase separated spontaneously to form condensates (shown for Wt in Fig. 3A and movie 4). Yet, phosphomimicking residues are less prone to interact with protein in the phase-separated condensates and are somewhat more solvated than the corresponding serine residues (Fig. 3B, Fig. S6). The aspartate side chains in 12D LCDs engage in partially compensatory interactions with arginines, showing that introduction of charged side chains gives rise to both stabilizing and destabilizing interactions in condensates. Importantly, our simulations (Fig. S6) are in line with previous studies that have highlighted the importance of aromatic sticker-sticker interactions in driving phase separation of prion-like domains and the TDP-43 LCD (Li et al., 2018; Martin et al., 2020).

To characterize the interactions of TDP-43 LCDs further, we performed atomistic molecular dynamics simulations of dense protein condensates (Fig. 3C, movie 5) assembled with hierarchical chain growth (Pietrek et al., 2020) to enhance the sampling of polymeric degrees of freedom. In microsecond dynamics with explicit solvent and a highly accurate description of molecular interactions (Robustelli et al., 2018), we again found serine residues in the $\mathrm{Wt}$ protein to be more prone to interact with other protein residues than interacting with solvent (Fig. 3D). By contrast, phosphomimicking aspartate side chains bind more water molecules and show an overall reduced tendency for protein-protein interactions, but with some 
compensating interactions in particular with arginines (Fig. 3D-F, Fig. S7A). Enhanced sidechain solvation is consistent across the 12 phosphomimetic substitution sites (Fig. S7B). The simulations thus suggest that individual small effects act together to modulate the phase separation behavior of TDP-43.

\section{C-terminal phosphomimetic substitutions do not impair nuclear import and RNA regulatory functions of TDP-43}

Next, we turned to cellular experiments to investigate how C-terminal TDP-43 phosphorylation affects the behavior and function of TDP-43 in cells. As TDP-43 hyperphosphorylation is found in the disease state, it seems possible that this PTM has detrimental effects on the protein and contributes to mislocalization and/or malfunction of TDP-43, thus driving neurodegeneration. To address this possibility, we expressed different TDP-43 variants (Wt, 12D, 12A) in HeLa cells and analyzed their intracellular localization, nuclear import and RNA processing functions. All three TDP-43 variants showed a predominantly nuclear localization (Fig. S8A), and nuclear import rates measured in a hormone-inducible reporter assay by live cell imaging (Hutten et al., 2020) were indistinguishable (Fig. 4A, B). To assess whether hyperphosphorylated TDP-43 shows functional impairments in RNA processing, we first assessed its ability to autoregulate its own levels (Avendano-Vazquez et al., 2012; Ayala et al., 2011). However, endogenous TDP-43 was downregulated to the same degree by all three TDP43 variants (Fig. 4C), indicating that hyperphosphorylated TDP-43 can normally bind to its own 3'UTR and autoregulate its own levels. In line with these findings, recombinant TDP-43 Wt, $12 \mathrm{D}$ and 12A showed comparable RNA binding in electrophoretic mobility shift assays (EMSAs) with in vitro transcribed RNA comprised of the autoregulatory TDP-43 binding site (Fig. 4D) or synthetic (UG) 12 RNA (Fig. S8B). Second, we examined splicing of two known TDP-43 splice targets that get mis-spliced upon loss of TDP-43 (Fiesel et al., 2012; Tollervey et al., 2011). After siRNA-mediated silencing of endogenous TDP-43 expression and reexpression of siRNA-resistant TDP-43 Wt, 12D or 12A (Fig. S8C), splicing of SKAR and Bim exon 3 were fully restored by all three TDP-43 variants (Fig. 4E), indicating normal function of phosphomimetic TDP-43 in splicing regulation. Together, our data suggest that C-terminal hyperphosphorylation is not responsible for cytosolic mislocalization or impaired RNA regulatory functions of TDP-43 in disease.

\section{Phosphorylation suppresses recruitment of TDP-43 into stress-induced membrane-less organelles (MLOs)}

Finally, we investigated how C-terminal TDP-43 phosphorylation affects TDP-43 condensation in cellular MLOs. First, we used a quantitative assay to measure SG association of recombinant proteins under controlled conditions in semi-permeabilized HeLa cells (Hutten and Dormann, 2020) (Fig. 5A). In line with our in vitro condensation experiments, increasing the number of phosphomimetic S-to-D substitutions caused a gradual decrease in SG association of TDP-43 (Fig. S9A, B). In vitro phosphorylated TDP-43 showed a similarly strong reduction in SG association as the $12 \mathrm{D}$ protein (Fig. 5B, C), demonstrating that the phosphomimetic substitutions and phospho-groups introduced by a kinase have similar effects on SG association of TDP-43. Second, we expressed the different TDP-43 variants in intact HeLa cells to analyze their recruitment into stress-induced MLOs. To this end, we silenced endogenous TDP-43 expression using siRNA (Fig. S9C) and then re-introduced siRNA-resistant myc-tagged TDP- 
$43 \mathrm{Wt}, 12 \mathrm{D}$ or 12A, thus avoiding oligomerization with endogenous TDP-43 via the N-terminal domain (Afroz et al., 2017). Short term oxidative stress treatment caused a partially cytosolic relocalization of TDP-43 and led to recruitment of TDP-43 Wt and 12A, but not the 12D mutant, into TIA-1-positive SGs (Fig. 5D, E). Similar results were obtained for nuclear import-deficient TDP-43 (Fig. 5F, G) that was strongly mislocalized to the cytoplasm due to point mutations in the nuclear localization signal (NLSmut) (Fig. S9D). Finally, we examined recruitment of TDP43 into arsenite-induced nuclear bodies (NBs) (Wang et al., 2020) and found that TDP-43 Wt and $12 \mathrm{~A}$ were readily recruited into stress-induced NBs, while the phosphomimetic 12D protein remained dispersed in the nucleoplasm (Fig. 5H-J). Taken together, phosphomimetic substitutions that mimic disease-linked phosphorylation of TDP-43 suppress the localization of TDP-43 in phase-separated MLOs that could be condensation sites for pathological TDP-43 aggregation.

\section{Phosphomimetic substitutions enhance TDP-43 solubility in Hela cells and primary neurons}

To further support the idea that phosphorylation enhances the solubility of TDP-43 and counteracts its aggregation propensity in cells, we overexpressed the different TDP-43 variants in HeLa cells and performed a biochemical fractionation into a RIPA-soluble (S) and RIPAinsoluble (I) fraction. Indeed, the $12 \mathrm{D}$ protein had a significantly higher S/I ratio compared to the Wt and 12A proteins (Fig. 6A, B). We also expressed GFP-tagged TDP-43 Wt, 12D, 12A or the corresponding NLS-mutant cytosolic versions in primary rat neurons and then probed for RIPA-insoluble high molecular weight material in a filter trap assay. Both the nuclear and the cytosolic 12D proteins showed a strong reduction in the amount of RIPA-insoluble TDP-43 in the transduced neurons (Fig. 6C). Confocal microscopy of transduced neurons revealed a completely dispersed localization of the NLS-mutant 12D protein, whereas TDP-43 Wt and, in particular, 12A showed a more granular, condensed pattern in the neuronal cytoplasm (Fig. 6D). Thus, we conclude that phosphomimetic substitutions mimicking disease-linked C-terminal hyperphosphorylation reduce TDP-43's tendency to condense and become insoluble in neurons and propose that TDP-43 phosphorylation might be a cellular response to counteract pathological TDP-43 aggregation. 


\section{Discussion}

C-terminal TDP-43 phosphorylation is a long-recognized pathological hallmark in ALS and FTD (Hasegawa et al., 2008; Inukai et al., 2008; Kametani et al., 2016; Neumann et al., 2009). Against previous expectations, we now show that this could be a protective rather than a pathogenic modification: We find that TDP-43 phosphorylation, and in particular phosphomimetic mutations mimicking the phosphorylation pattern in ALS/FTD (Hasegawa et al., 2008; Kametani et al., 2016), strongly suppress TDP-43 phase separation and aggregation both in vitro and in cells. Our data is in line with two previous studies that examined C-terminal fragments of TDP-43 with phosphomimetic 2D or 5D/E mutations and observed a reduced aggregation propensity and toxicity in cell lines and Drosophila (Brady et al., 2011; Li et al., 2011). Based on a recent cryo-EM structure of TDP-43 LCD fibrils, several C-terminal serines are buried inside the fibril structure (Li et al., 2021), hence phosphorylation could disrupt the amyloid fibril structure, in line with our experimental findings. We therefore propose that TDP43 phosphorylation might be a protective cellular mechanism that counteracts aberrant TDP-43 phase transitions and renders TDP-43 more dynamic and liquid-like by reducing C-terminal LCD-LCD interactions through negatively charged, highly hydrated phospho-groups.

What triggers TDP-43 phosphorylation and how phosphorylation or the hyperphosphorylated protein is removed is still unknown. Interestingly, we and others previously found that C-terminal TDP-43 phosphorylation follows TDP-43 insolubility, suggesting that phosphorylation arises downstream of TDP-43 aggregation (Brady et al., 2011; Dormann et al., 2009). We speculate that hyperphosphorylated soluble TDP-43 is rapidly cleared, e.g. by the proteasome, and therefore is not detected under normal conditions. Under conditions of proteostasis impairment, e.g. in the aging brain and specifically in ALS/FTD patients (Hipp et al., 2019; Yerbury et al., 2020), clearance mechanisms might be impaired, leading to accumulation of hyperphosphorylated, polyubiquitinated TDP-43 that then may aggregate due to elevated protein concentration, additional PTMs, or aberrant interactions with other cellular molecules/organelles.

Several other studies on TDP-43 phosphorylation at first glance contrast our findings. Overexpression of various TDP-43 kinases in cell or animal models was shown to promote TDP-43 aggregation and neurotoxicity (Choksi et al., 2014; Liachko et al., 2014; Nonaka et al., 2016; Taylor et al., 2018). Based on these studies, inhibition of TDP-43 phosphorylation by kinase inhibitors has been proposed as a potential therapeutic strategy for ALS (Liachko et al., 2013; Martinez-Gonzalez et al., 2020; Salado et al., 2014). A possible explanation for the discrepant findings could be that kinase overexpression has pleiotropic effects that may cause TDP-43 aggregation and neurotoxicity independent of TDP-43 phosphorylation. Our data exclude such indirect effects, as they rely on experiments with purified components and defined phosphomimetic constructs rather than modulation of kinase levels/activity. Furthermore, our results suggest that beneficial effects seen with kinase inhibitors are likely not the direct consequence of reduced TDP-43 phosphorylation, but rather mediated by other mechanisms.

An alternative scenario that we cannot exclude is that reduced TDP-43 condensation due to hyperphosphorylation may have negative consequences by disturbing essential functions of TDP-43 that depend on its capacity to phase separate or solidify, e.g. certain DNA/RNA processing steps or recruitment of TDP-43 into cytoprotective NBs (Wang et al., 2020) or other 
MLOs. In support of this hypothesis, a deep mutagenesis study recently found that aggregating TDP-43 variants decrease toxicity in yeast, whereas dynamic, liquid-like variants enhance toxicity (Bolognesi et al., 2019), so further work is needed to investigate this possible scenario. However, our data clearly show that the core RNA processing functions (autoregulation and splicing regulation), RNA-binding and nuclear localization/import of TDP-43 are not affected by C-terminal hyperphosphorylation and therefore do not depend on TDP-43's phase separation and solidification capacity.

Of note, abnormal PTMs are a common theme in neurodegenerative disorders, e.g. Tauopathies linked to pathological Tau aggregation (Alquezar et al., 2020; Morris et al., 2015). Interestingly, even though hyperphosphorylation is generally believed to trigger Tau aggregation, site-specific phosphorylation in the microtubule-binding region of Tau was recently shown to inhibit, rather than promote Tau fibrillization and seeding (Haj-Yahya et al., 2020). We now show that C-terminal TDP-43 phosphorylation as detected on ALS/FTD inclusions has a similar inhibitory effect on TDP-43 aggregation, underscoring the idea that aberrant PTMs detected on pathological inclusions may not necessarily all be drivers of protein aggregation, but could also have protective, anti-aggregation effects that are later-on overruled by other pathogenic mechanisms. 


\section{Acknowledgments}

We thank Bettina Schmid, Sören von Bülow and Zakarya Benayad for insightful discussions and Ignasi Forné for technical support and discussion. We acknowledge support by the Core Facilities Proteomics, Bioimaging and Bioinformatics of the BMC Munich and thank Peter Becker and Michael Kiebler for infrastructure and access to the spinning disc confocal microscope (DFG, INST 86/1581-1 FUGG). This work was supported by the Deutsche Forschungsgemeinschaft (DFG, German Research Foundation) by project DO1804/4-1 within SPP2191 - ID 402723784 (to D.D.), the Munich Cluster for Systems Neurology (EXC2145 SyNergy - ID 390857198 to D.D. and D.E.) and the NOMIS foundation (D.E.). G.H. acknowledges financial support from the German Research Foundation (CRC 902: Molecular Principles of RNA Based Regulation) and the Max Planck Society. L.S.S. acknowledges support by ReALity - Resilience, Adaptation and Longevity, M3ODEL and Forschungsinitiative des Landes Rheinland-Pfalz.

\section{Author contributions}

Conceptualization, D.D., L.G.S.; Methodology, all authors; Investigation, L.G.S., F.S., S.H., H.R., E.L.S., J.G., L.S.S.; Resources, D.D., G.H., L.S.S., D.E., V.D.; Writing - Original Draft, D.D., L.G.S.; Writing - Review and Editing, all authors; Visualization, L.G.S., F.S., S.H., H.R., E.L.S., J.G., L.S.S.; Supervision, D.D., G.H., D.E., V.D., L.S.S.; Project Administration, D.D.; Funding Acquisition, D.D., D.E., G.H., L.S.S., V.D.

\section{Competing interests}

Authors declare no competing interest.

\section{Data and materials availability}

This study includes no data deposited in external repositories. 


\section{Materials and Methods}

\section{$\underline{\text { cDNA constructs }}$}

\section{Bacterial expressing constructs}

TDP-43 carrying mutations in serine 409 and 410, either to aspartate (2D) or alanine (2A) were generated by site-directed mutagenesis using Q5 high fidelity DNA polymerase (NEB) using primers containing the mutations S409D/410D and S409A/410A and pJ4M TDP-43-TEVMBP-His 6 vector as a template. Expression constructs with 5 or 12 serine substitutions $(5 \mathrm{D}$, 5A, 12D and 12A) were generated using synthetic double stranded DNA fragments (gBlocks Gene Fragments, IDT) containing the respective mutations, cloned into PstI and XhoI sites of the pJ4M TDP-43-TEV-MBP-His6-backbone.

\section{Mammalian expressing constructs}

To generate an expressing construct coding for Myc-hTDP-43, the coding sequence of hTDP43 was PCR amplified from pEGFP-C1-hTDP-43 (Ederle et al., 2018), including a Myc coding sequence in the forward PCR primer, and cloned into a pcDNA5-FRT-TO-backbone using XhoI and BamHI restriction sites. Note, that the hTDP-43 template includes a resistance to TARDBPHSS118765 siRNA (Invitrogen) used to silence endogenous TDP-43 (see below "siRNA-mediated knockdown of TDP-43"). For generation of the TDP-43 12D and 12A constructs, synthetic gBlocks (IDT) harboring the respective mutations were previously cloned into the NdeI and BamHI sites of the pEGFP-C1-hTDP-43 vector. In constructs carrying mutations in the NLS of TDP-43 (mNLS), amino acids 82-84 as well as 95,97,98 were exchanged for alanine (pEGFP-hTDP-43 double-mNLS). Then, the mNLS region was transferred from the pEGFP-TDP-43 double-mNLS template to the pcDNA5-FRT-TO-MychTDP-43, 12D and 12A vectors via the restriction enzymes XhoI and NdeI. To generate the $\mathrm{GCR}_{2}-\mathrm{GFP}_{2}-\mathrm{TDP}-43 \mathrm{12D}$ and $12 \mathrm{~A}$ constructs, the respective coding sequences were PCR amplified and inserted into $\mathrm{GCR}_{2}-\mathrm{GFP}_{2}$-backbone using EcoRV and BamHI. To allow for lentiviral packaging and subsequent neuronal transduction, coding sequences of TDP-43 Wt, $12 \mathrm{D}$ and $12 \mathrm{~A}$ were subcloned into the FhSynW backbone in frame with mEGFP (May et al., 2014).

\section{Cell culture, transfection and stress treatment}

HeLa cells were grown in DMEM high glucose GlutaMAX (Invitrogen) supplemented with $10 \%$ fetal bovine serum (FBS) and $10 \mu \mathrm{g} / \mathrm{ml}$ gentamicin and incubated in a humidified chamber with $5 \% \mathrm{CO}_{2}$ at $37^{\circ} \mathrm{C}$. cDNA transfections were performed using Lipofectamine 2000 (Thermo) in culture medium without gentamicin and medium was exchanged after 4 to 6 hours to avoid cellular stress by the transfection reagent. Note, that for equal transfection efficiency different amounts of DNA were transfected for the different constructs (For 12D: 100\%; for Wt and 12A: $75 \%+25 \%$ empty vector DNA). For immunostaining cells were fixed after $\sim 24 \mathrm{~h}$. Hydrogen peroxide $\left(\mathrm{H}_{2} \mathrm{O}_{2}\right)(1 \mathrm{mM})$ treatment was carried out for $2 \mathrm{~h}$, MG132 $(10 \mu \mathrm{M})$ treatment for 2.5$3 \mathrm{~h}$ and sodium arsenite $(0.5 \mathrm{mM})$ treatment for $45 \mathrm{~min}$.

\section{Neuronal cell culture and lentiviral packaging}

Primary hippocampal and cortical neuronal cultures were prepared from embryonic day 19 rats as described in detail previously (Guo et al., 2018). In brief, neocortex and hippocampus were 
dissected, followed by enzymatic dissociation and gentle trituration. For immunofluorescence experiments, hippocampal neurons $(85000 \mathrm{cells} / \mathrm{ml})$ were plated on poly-D-lysine-coated glass coverslips (VWR) in 12-well plates (Thermo Fisher) and cultured in Neurobasal medium (Thermo Fisher) supplemented with 2\% B27 (Thermo Fisher), 1\% Penicillin-Streptomycin (Thermo Fisher), $0.5 \mathrm{mM}$ L-glutamine (Thermo Fisher) and $12.5 \mu \mathrm{M}$ glutamate (Thermo Fisher). Both, cortical and hippocampal neurons, were transduced on day in vitro (DIV) 5.

Cortical neurons $(250000 \mathrm{cells} / \mathrm{ml}$ ) used for filter trap assays were plated on poly-D-lysinecoated 6-well plates and cultured in Neurobasal medium containing 2\% B27, 1\% PenicillinStreptomycin and $0.5 \mathrm{mM}$ L-glutamine.

Lentiviral packaging was performed by seeding HEK293FT cells (Thermo Fisher) of low passage number into three $10 \mathrm{~cm}$ dishes per construct $\left(5 \times 10^{6}\right.$ cells/dish). Cells were plated in DMEM, high glucose, GlutaMAX (Thermo Fisher) supplemented with 10\% FBS (Sigma), 1\% Penicillin-Streptomycin (Thermo Fisher) and 1\% Non-Essential Amino Acids (Thermo Fisher). On the following day, cells were co-transfected with $18.6 \mu \mathrm{g}$ transfer vector (FhSynW-mEGFPhTDP-43, FhSynW-mEGFP-hTDP-43 (12D), FhSynW-mEGFP-hTDP-43 (12A), FhSynWmEGFP-hTDP-43-mNLS, FhSynW-mEGFP-hTDP-43-mNLS (12D), FhSynW-mEGFPhTDP-43-mNLS (12A)), $11 \mu \mathrm{g}$ pSPAX2 and $6.4 \mu \mathrm{g}$ pVSVG using Lipofectamine 2000 (Thermo Fisher). The transfection media was replaced by plating media supplemented with 13 $\mathrm{mg} / \mathrm{mL}$ bovine serum albumin (BSA, Sigma) on the next day. Lentivirus from the cell supernatant was collected $24 \mathrm{~h}$ later by ultracentrifugation with a Sw28 rotor (Beckman Coulter; 22,000 rpm, $\left.2 \mathrm{~h}, 4^{\circ} \mathrm{C}\right)$. Finally, lentiviral particles were resuspended in Neurobasal media (Thermo Fisher), stored at $-80^{\circ} \mathrm{C}$ and used for lentiviral transduction by adding to neuronal culture media upon thawing. Neurons were kept in culture for 4 additional days after transduction on DIV5 (DIV5+4).

\section{$\underline{\text { Recombinant protein expression and purification }}$}

TDP-43-TEV-MBP-His6

All TDP-43-MBP-His6 variants were purified according to (Wang et al., 2018) with minor adaptations. First, expression of proteins was performed in E. coli BL21-DE3 Rosetta 2 using $0.5 \mathrm{mM}$ IPTG and grown overnight at $16^{\circ} \mathrm{C}$. Next, cells were resuspended in lysis buffer (20 $\mathrm{mM}$ Tris $\mathrm{pH} 8,1 \mathrm{M} \mathrm{NaCl}, 10 \mathrm{mM}$ imidazole, $10 \%(\mathrm{v} / \mathrm{v})$ glycerol, $4 \mathrm{mM} \beta$-mercaptoethanol and $1 \mu \mathrm{g} / \mathrm{ml}$ each of aprotinin, leupeptin hemisulfate and pepstatin A) supplemented with 0.1 $\mathrm{mg} / \mathrm{ml}$ RNase A, and lysed using lysozyme and sonication. Subsequently, the protein was purified by Ni-NTA agarose (Qiagen) and eluted with lysis buffer containing $300 \mathrm{mM}$ imidazole. For all TDP-43-MBP-His6 variants a final size exclusion chromatography (SEC) (Hiload 16/600 Superdex $200 \mathrm{pg}$, GE Healthcare) purification step was performed in purification buffer $(20 \mathrm{mM}$ Tris $\mathrm{pH} 8,300 \mathrm{mM} \mathrm{NaCl}, 10 \%$ (v/v) glycerol supplemented with 2 $\mathrm{mM}$ TCEP), in order to separate TDP-43-MBP-His 6 from protein aggregates and contaminants. Purified monomeric TDP-43-MBP-His6 was collected by pooling the fractions corresponding to peak B in the SEC profile (figure S1D). All purified proteins were concentrated using Amicon ultra centrifugal filters and then flash frozen and stored at $-80^{\circ} \mathrm{C}$. To determine protein concentration, absorbance at $280 \mathrm{~nm}$ was measured using the respective extinction coefficient ( $\varepsilon$ ) predicted by the ProtParam tool. Additionally, for all purified proteins, the A260/280 ratio was determined and found to be between 0.5-0.7. 
$C K 1 \delta$

The kinase domain of CSNK1D was expressed as an N-terminal MBP-tagged fusion in E. coli Rosetta 2 cells, co-expressing $\lambda$-phosphatase to guarantee a completely unphosphorylated protein. The cells were grown to an OD of 0.45 and subsequently the temperature was reduced to $18^{\circ} \mathrm{C}$. Then the cells were induced (generally at OD 0.7-0.8) with $0.5 \mathrm{mM}$ IPTG and expression was performed overnight. Cells were harvested and resuspended in AC-A buffer (25 mM Bis-Tris, $500 \mathrm{mM} \mathrm{NaCl}, 10 \mathrm{mM} \beta$-mercaptoethanol, $\mathrm{pH}$ 7.0), supplemented with DNAse, RNAse, lysozyme and protease inhibitor cocktail (selfmade) for cell disruption. Lysis was done by sonication on ice ( $5 \times 30 \mathrm{~s}$ with breaks of $1 \mathrm{~min}$ between each pulse). Cell debris was pelleted by centrifugation (SS34 rotor, 17000 r.p.m., $30 \mathrm{~min}$ ). The supernatant was filtered and subsequently loaded on a Dextrin Sepharose column (cytiva), previously equilibrated with ACA buffer. The column was washed for 5 column volumes with AC-A buffer. Elution was done with MBP-B buffer (25 mM Bis-Tris, $500 \mathrm{mM} \mathrm{NaCl,} 10 \mathrm{mM} \beta$-mercaptoethanol, $20 \mathrm{mM}$ Maltose, $\mathrm{pH}$ 7.0). The eluted protein was subject to TEV protease cleavage overnight at $4{ }^{\circ} \mathrm{C}$. On the next day the buffer was exchanged to IEX-A buffer $(25 \mathrm{mM}$ Bis-Tris, $50 \mathrm{mM} \mathrm{NaCl}, 10$ mM. $\beta$-mercaptoethanol) by ultra-filtration (Amicon Ultra-15 $30 \mathrm{kDa}$, Merck Millipore) and subject to cation-exchange chromatography by a linear to IEX-B buffer ( $25 \mathrm{mM}$ Bis-Tris, 500 $\mathrm{mM} \mathrm{NaCl}, 10 \mathrm{mM}$. $\beta$-mercaptoethanol). Eluted protein was concentrated and gel-filtered over a Superdex 75 (cytiva) into SEC buffer (25 mM Bis-Tris, $50 \mathrm{mM} \mathrm{NaCl}, 10 \mathrm{mM} \mathrm{MgCl} 2,1 \mathrm{mM}$ DTT). Fractions were collected, concentrated and aliquots of $200 \mu 1$ were flash frozen and stored at $-80^{\circ} \mathrm{C}$ until use.

His6-TEV protease

His6-TEV protease expression and purification was performed as described in (Hutten et al., 2020).

\section{In vitro phosphorylation}

TDP-43-MBP-His6 was in vitro phosphorylated with $\mathrm{CK} 1 \delta$ and $200 \mu \mathrm{M}$ Adenosine triphosphate (ATP) in phosphorylation buffer (50 mM Tris- $\mathrm{HCl}, \mathrm{pH} 7.5,10 \mathrm{mM} \mathrm{MgCl} 2,1 \mathrm{mM}$ DTT) for $2 \mathrm{~h}$ at RT, using a 2-fold molar excess of TDP-43-MBP-His6 over CK1 $\delta$. Subsequently, the reaction was used for sedimentation and SG association assays. As negative controls, either the kinase or the ATP was omitted and also included as controls in subsequent assays.

Enzymatic digestion, enrichment for phosphopeptides and mass spectrometric analysis TDP-43-MBP-His6 was in vitro phosphorylated as described above, separated on a $10 \%$ SDSPAGE gel and visualized by Coomassie staining. The gel band corresponding to the phosphorylated TDP-43-MBP-His6 was excised and destained twice for $30 \mathrm{~min}$ at $37{ }^{\circ} \mathrm{C}$ with $50 \%$ acetonitrile in $50 \mathrm{mM}$ Tris- $\mathrm{HCl}, \mathrm{pH}$. The gel piece was dehydrated with $100 \%$ acetonitrile, reduced and alkylated, and finally digested overnight at $37^{\circ} \mathrm{C}$ with $375 \mathrm{ng}$ Trypsin (Promega). The peptides were extracted from the gel twice using $100 \mu 1$ of $50 \%$ acetonitrile and $0.25 \%$ TFA buffer. Both extractions were merged and evaporated in a vacuum evaporator. In order to enrich the phosphopeptides, $10 \mu 1$ of $0.5 \mathrm{mg} / \mu 1 \mathrm{TiO} 2$ beads (GL Sciences Cat.No: 
5010-21315) in loading buffer ( $80 \%$ ACN, 5\% TFA, $1 \mathrm{M}$ Glycolic acid) were added to the dried samples in a ratio of $0.3 \mathrm{mg}$ of beads to $5 \mathrm{pmol}$ of protein. Samples were incubated for $10 \mathrm{~min}$ at RT on a shaker at $2000 \mathrm{rpm}$ and spun down at $1200 \mathrm{rpm}$ for $1 \mathrm{~min}$. The supernatant was removed and kept for further analysis, while beads were sequentially washed with loading buffer, washing buffer 1 ( $80 \%$ ACN, 1\% TFA) and washing buffer 2 (10\% ACN, $0.2 \%$ TFA). Next, the beads were dried in the hood for 10 min and resuspended with $50 \mu 1$ elution buffer (28\% ammonia solution in $\mathrm{H}_{2} \mathrm{O}$ ). Finally, the samples were speed vacuum evaporated and resuspended with $15 \mu 1$ 0,1 \% FA. For LC-MS purposes, desalted peptides were injected in an Ultimate 3000 RSLCnano system (Thermo) and separated in a $25-\mathrm{cm}$ analytical column $(75 \mu \mathrm{m}$ ID, $1.6 \mu \mathrm{m} \mathrm{C18}$, IonOpticks) with a 30 -min gradient from 3 to $30 \%$ acetonitrile in $0.1 \%$ formic acid. The effluent from the HPLC was directly electrosprayed into a Qexactive HF (Thermo) operated in data dependent mode to automatically switch between full scan MS and MS/MS acquisition. Survey full scan MS spectra (from m/z 300-1600) were acquired with resolution $\mathrm{R}=60,000$ at $\mathrm{m} / \mathrm{z} 400$ (AGC target of $3 \times 106$ ). The 10 most intense peptide ions with charge states between 2 and 5 were sequentially isolated to a target value of $1 \times 10^{5}$ with resolution $\mathrm{R}=15,000$ and isolation window $1.6 \mathrm{Th}$ and fragmented at $27 \%$ normalized collision energy. Typical mass spectrometric conditions were: spray voltage, $1.5 \mathrm{kV}$; no sheath and auxiliary gas flow; heated capillary temperature, $250^{\circ} \mathrm{C}$; ion selection threshold, 33.000 counts.

\section{Fluorescent labeling of purified TDP-43}

TDP-43-MBP-His6 variants were labeled with Alexa Fluor 488 C5 maleimide (Thermo Fisher) at a low ( 0.01-0.05) labelling efficiency in order to avoid interference with condensate formation. Labeling was performed according to the manufacture's protocol using a 1:100 or 1:20 protein:fluorescent dye mole ratio. Briefly, the Alexa Fluor reagent, previously dissolved in DMSO, was mixed with the protein and kept in the dark for $2 \mathrm{~h}$ at RT. Excess dye was removed by consecutive washes with TDP-43 purification buffer using Amicon ultra centrifugal filters. Subsequently, labelled protein was used for spinning disc confocal microscopy, FRAP and aggregation assays, respectively.

\section{$\underline{\text { In vitro phase separation and aggregation assays }}$}

\section{Sedimentation assay}

For sedimentation analysis, $1 \mu \mathrm{M}$ TDP-43-TEV-MBP-His6 variants or in vitro phosphorylated TDP-43-TEV-MBP-His6 was cleaved by addition of $20 \mu \mathrm{g} / \mathrm{ml}$ His6-TEV protease in $50 \mu 1$ or $25 \mu 1$ Hepes buffer (20 mM Hepes, pH 7.5, $150 \mathrm{mM} \mathrm{NaCl}, 1 \mathrm{mM}$ DTT), respectively, to remove the MBP-His 6 tag and induce phase separation. Samples were incubated for $60 \mathrm{~min}$ at $30{ }^{\circ} \mathrm{C}$, followed by centrifugation for $15 \mathrm{~min}$ at $21,000 \mathrm{~g}$ at $4^{\circ} \mathrm{C}$ to pellet the formed condensates. Equal amounts of supernatant (S) and condensate (C) fractions were loaded onto an SDS-PAGE gel and TDP-43 was detected by Western Blot (rabbit TDP-43 N-term, Proteintech, Cat.No: 107822-AP).

\section{Microscopic condensate assay}

For all microscopic condensate assays Uncoated $\mu$-Slide 18 Well - Flat chambers (Cat.No: 81821, Ibidi) were pretreated with $10 \%$ Pluronics F-127 solution for $1 \mathrm{~h}$ and 5 times washed with MilliQ water. The water remained in the chamber until just before the experiment, as described in (Ceballos et al., 2018). 
Purified TDP-43-TEV-MBP-His6 variants were buffer exchange to Hepes buffer or phosphate buffer $\left(20 \mathrm{mM} \mathrm{Na} 2 \mathrm{HPO}_{4} / \mathrm{NaH}_{2} \mathrm{PO}_{4}, \mathrm{pH} 7.5,150 \mathrm{mM} \mathrm{NaCl}, 2.5 \%\right.$ glycerol, $1 \mathrm{mM}$ DTT). Proteins were then centrifuged at $21,000 \mathrm{~g}$ for $10 \mathrm{~min}$ at $4{ }^{\circ} \mathrm{C}$ to remove any preformed protein precipitates. For condensates formation, the reaction was setup directly in Pluronicscoated $\mu$-Slide 18 Well - Flat chambers, where proteins were diluted to the indicated concentrations and phase separation was induced by addition of $100 \mu \mathrm{g} / \mathrm{ml}$ His6-TEV protease at RT. After $\sim 20$ min, imaging was performed by bright field microscopy using a widefield microscope.

For fusion events and FRAP analysis, condensates were formed directly in Pluronicscoated $\mu$-Slide 18 Well - Flat chambers as described above using $20 \mu \mathrm{M}$ of each Al.488-labelled TDP-43 protein variants (Wt, 5D, 12D) in Hepes buffer and incubated for $10 \mathrm{~min}$ at RT before imaging. Note that experiments were performed until maximally $1 \mathrm{~h}$ after adding the TEV protease, in order to avoid in vitro aging of condensates.

\section{Turbidity assay}

Phase separation of TDP-43-TEV-MBP-His6 variants was induced as described above for the microscopic condensate assay. Reactions of $20 \mu \mathrm{l}$ samples were prepared at the indicated concentrations in 384-well plates and incubated for $30 \mathrm{~min}$ at RT after adding TEV protease. Subsequently, a BioTek Power Wave HT plate reader was used to measure turbidity at $600 \mathrm{~nm}$. Turbidity measurements were performed in triplicates.

\section{Semi-denaturing Detergent Agarose Gel Electrophoresis (SDD-AGE)}

SDD-AGE experiments were performed based on protocols published by (French et al., 2019) and (Halfmann and Lindquist, 2008). First, $2 \mu \mathrm{M}$ purified TDP-43-MBP-His6 variants (WT, $5 \mathrm{D}, 12 \mathrm{D}, 12 \mathrm{~A}$ ) were set up in low binding tubes (Eppendorf) in $35 \mu 1$ aggregation buffer (50 $\mathrm{mM}$ Tris $\mathrm{pH} 8.0,250 \mathrm{mM} \mathrm{NaCl}, 5 \%$ glycerol, $5 \%$ sucrose, $150 \mathrm{mM}$ imidazole $\mathrm{pH} 8.0$ ) and supplemented with 1x protease inhibitor (Sigma). Samples were shaken for $30 \mathrm{~min}$ at $1000 \mathrm{rpm}$ at RT $\left(\sim 22^{\circ} \mathrm{C}\right)$ and then incubated at RT for the indicated time period. $5 \mu$ of each sample was collected and diluted in SDD-AGE buffer (40 mM Tris- $\mathrm{HCl} \mathrm{pH}$ 6.8, 5\% Glycerol, $0.5 \%$ SDS, $0.1 \%$ bromphenol-blue) and analyzed by SDD-AGE by horizontal $1.5 \%$ agarose gel electrophoresis (gel: $1.5 \%$ agarose in $20 \mathrm{mM}$ Tris, $200 \mathrm{mM}$ Glycine and $0.1 \%$ SDS) in running buffer (60 mM Tris, $20 \mathrm{mM}$ Acetate, $200 \mathrm{mM}$ glycine, $1 \mathrm{mM}$ EDTA and $0.1 \% \mathrm{SDS}$ ) for $\sim 6 \mathrm{~h}$ at $60 \mathrm{~V}$. Detection of TDP-43 monomers, oligomers and high molecular weight species was performed after overnight capillary transfer in TBS (50 mM Tris $\mathrm{pH} 7.6,150 \mathrm{mM} \mathrm{NaCl})$ to a nitrocellulose membrane and by standard Western Blot using rabbit anti TDP-43 N-term antibody (Proteintech, Cat.No: 10782-2-AP).

\section{Formation of Alexa 488-labelled TDP-43 aggregates}

In order to visualize TDP-43 (wt, 5D, 12D and 12A) aggregates formed under the above described assay conditions, $10 \mu \mathrm{M}$ A1.488-labeled TDP-43-MBP-His6 was set up in low binding tubes (Eppendorf) in aggregation buffer and incubated with or without $100 \mu \mathrm{g} / \mathrm{ml} \mathrm{His} 6$ TEV protease. Samples were shaken at $1000 \mathrm{rpm}$ at RT for $30 \mathrm{~min}$ and then transferred into a 384-well black plate (Greiner Bio-One), incubated at RT and imaged by confocal microscopy after 2, 8 and $24 \mathrm{~h}$. 


\section{Cellular TDP-43 solubility assays}

\section{Fractionation in RIPA-Benzonase buffer}

HeLa cells $\left(\sim 1 \times 10^{6}\right)$ were washed twice in PBS, harvested by scraping and pelleted at $3600 \mathrm{rpm}$ for $5 \mathrm{~min}$. Cell pellets were incubated on ice for $15 \mathrm{~min}$ in $200 \mu 1$ RIPA buffer $(50 \mathrm{mM}$ Tris$\mathrm{HCl}, \mathrm{pH} 8.0,150 \mathrm{mM} \mathrm{NaCl}, 1 \% \mathrm{NP}-40,0.5 \%$ deoxycholate, $0.1 \%$ SDS) with freshly added $1 \mathrm{x}$ protease inhibitor cocktail (Sigma), 1x phosphatase inhibitors (final concentration: $10 \mathrm{mM} \mathrm{NaF}$, $1 \mathrm{mM} \beta$-glycerophosphate, $1 \mathrm{mM} \mathrm{Na3VO4)}$ and $0.05 \mathrm{unit} / \mu 1$ Benzonase (Sigma). Samples were sonicated in a BioRuptorPico (Diagenode) for $45 \mathrm{sec}$ and $20 \mu \mathrm{l}$ of sample was collected as "Input". The remaining sample was then centrifuged at $13,000 \mathrm{~g}$ for $30 \mathrm{~min}$ at $4^{\circ} \mathrm{C}$. The resulting supernatants $(\mathrm{S})$ were collected and the remaining pellets were washed in RIPA buffer with inhibitors, resonicated for $45 \mathrm{sec}$ and recentrifuged for $30 \mathrm{~min}$ at $4{ }^{\circ} \mathrm{C}$ at $13,000 \mathrm{~g}$. Finally, the RIPA insoluble pellets (I) were resuspended in $36 \mu$ Urea buffer ( $7 \mathrm{M}$ urea, $2 \mathrm{M}$ thiourea, 4\% CHAPS, $30 \mathrm{mM}$ Tris-HCl, $\mathrm{pH} 8.5$ ) and sonicated. All samples were supplemented with 4x

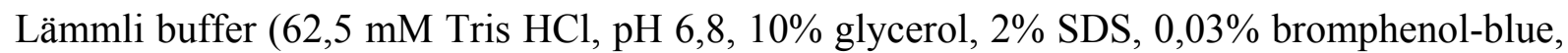
$143 \mathrm{mM} \beta$-Mercaptoethanol) and Input and Supernatant (S) samples were boiled prior to SDSPAGE and Western Blot against TDP-43 (rabbit anti TDP-43 C-term, Proteintech, Cat. No:12892-1-AP) and Myc (mouse anti-myc 9E10 antibody, Helmholtz Center Munich). Note that for detection reasons, the (I) fractions were $4 \mathrm{x}$ more concentrated than the (S) fractions, so they are represented in a 1:5 ratio.

\section{Filter trap assay}

Cortical neurons expressing the indicated GFP-tagged TDP-43 variants (DIV5 +4 days expression) were washed two times with PBS and lysed on ice in RIPA buffer (50 mM Tris$\mathrm{HCl}, \mathrm{pH} 8.0,150 \mathrm{mM} \mathrm{NaCl}, 1 \% \mathrm{NP}-40,0.5 \%$ deoxycholate, $0.1 \%$ SDS) freshly supplemented with 1x protease inhibitor cocktail (Sigma), 1x phosphatase inhibitor cocktail (Sigma) and 0.125 Units $/ \mu \mathrm{L}$ Benzonase (Sigma) for $20 \mathrm{~min}$. Cell lysates were collected and centrifuged at $1,000 \mathrm{~g}, 4^{\circ} \mathrm{C}$ for $30 \mathrm{~min} .2 / 3$ of the resulting supernatant (RIPA-insoluble fraction) was filtered through a nitrocellulose membrane $(0.2 \mu \mathrm{M}$ pore size, Merck) using a filter trap slot blot (Hoefer Scientific Instruments). After washing with PBS for three times, membranes were blocked for $1 \mathrm{~h}$ with $2 \%$ I-Block (Thermo Fisher) prior to immuno-detection with mouse antiGFP (UC Davis/NIH Neuromab Facility, Cat.No: N86/8) and rabbit anti Calnexin antibody (Enzo Life Sciences, Cat.No: ADI-SPA-860). The remaining 1/3 of the lysates was diluted with $3 x$ loading buffer (200 mM Tris-HCl pH 6.8, 6\% SDS, 20\% glycerol, $0.1 \mathrm{~g} / \mathrm{ml} \mathrm{DTT,} 0.1 \mathrm{mg}$ Bromophenol Blue), boiled at $95^{\circ} \mathrm{C}$ and used for subsequent standard Western Blot analysis.

\section{$\underline{\text { Multi scale simulations }}$}

\section{Coarse-grained MD simulations}

Coarse-grained MD simulations with explicit solvent to investigate protein phase separation and phase-separated protein condensates were run with a rescaled version of the Martini forcefield (Marrink et al., 2007; Monticelli et al., 2008) as described by (Benayad et al., 2021). A similar approach was shown to describe the conformational ensembles of proteins with disordered domains very well (Larsen et al., 2020; Martin et al., 2021), and we recently showed that such approaches can be extended to simulations of liquid-liquid phase separation of disordered proteins (Benayad et al., 2021). Protein-protein interactions were thus scaled to 0.8 
of the default value. Chloride and sodium ions were added to neutralize the system in simulations of $\mathrm{Wt}$ and $12 \mathrm{D}$ proteins. $10 \%$ of the water beads were replaced by WF anti-freeze beads. Coarse-grained simulations were run with GROMACS 2018 (Abraham et al., 2015). Simulations boxes measured 450 x 450 x 600 Å. Simulations systems were energy minimized and equilibrated in MD simulations with and without position restraints. $118 \mathrm{Wt}$ and $12 \mathrm{D} \mathrm{C}$ terminal LCDs (aa. 261 - 414) were simulated for $20 \mu$ s each. The coarse-grained simulations systems consist of roughly one million particles. Equations of motions were integrated with a 20 fs time step. Simulations were conducted in the NPT ensemble at 1 bar and $300 \mathrm{~K}$ using the Parrinello-Rahman barostat (Parrinello and Rahman, 1981) and the Bussi-Donadio- Parrinello velocity-rescaling thermostat (Bussi et al., 2007).

Note that in the coarse-grained approach we employed, four atoms are typically group together to a coarse-grained particle. E.g., a coarse-grained water molecule would correspond to four water molecules in an atomistic simulation.

\section{Atomistic MD simulations}

Hierarchical chain growth (HCG) (Pietrek et al., 2020) enables us to generate statistically independent and chemically-meaningful conformations of a biomolecular condensate with atomic resolution, which serve as starting points for atomistic MD simulations. Atomicresolution models of clusters of the C-terminal disordered domain of TDP-43 (aa. 261 - 414) were generated for both $\mathrm{Wt}$ protein and the 12D mutant. To assemble disordered proteins into a condensate, we first assemble pairs of disordered domains, then pairs of pairs, pairs of quadruplets, and so forth, following the logic set out in (Pietrek et al., 2020). HCG Monte Carlo manifestly satisfies detailed balance and thus we generate representative ensembles. Finally, we arrive at densely packed disordered domains, while retaining atomic resolution at each modeling step. Periodic boundary conditions were employed during the assembly.

Clusters of Wt and 12D LCDs were solvated in a $150 \AA$ x $150 \AA$ x $150 \AA$ simulation box, the system charge was neutralized and $150 \mathrm{mM} \mathrm{NaCl}$ was added. We employed the Amberdisp protein force field developed by Robustelli and et al. (Robustelli et al., 2018), including the modified TPIP4P-D water model (Piana et al., 2015) that accompanies the Amber-disp protein force field. Temperature was maintained at $300 \mathrm{~K}$ by the Bussi-Donadio-Parrinello velocity-rescaling thermostat (Bussi et al., 2007). We employed the Parrinello-Rahman barostat (Robustelli et al., 2018) to set the pressure to 1 bar. Equations of motions were integrated with a 2 fs time step. Production simulations were prepared by energy minimization with and without soft-core potentials. To start production simulations, we equilibrated the atomistic simulations systems, running at least 5000 steps with a 1 fs time step and position restraints and for $1.5 \mathrm{~ns}$ with a 2 fs time step also with position restraints. Equilibrium simulations of the clusters of the disordered domains were conducted with GROMACS 2019 (Abraham et al., 2015). For both wild-type and 12D clusters of 32 chains with 154 residues were simulated for $1 \mu$ s with two repeats each started from independently generated HCG structures.

Simulations were analyzed with the MDAnalysis (Gowers et al., 2016; Michaud-Agrawal et al., 2011) and the MDtraj Python libraries (McGibbon et al., 2015). Contact analysis was performed with the Contact Map Explorer Python library (https://github.com/dwhswenson/contact_map). 
To analyze import of $\mathrm{GCR}_{2}-\mathrm{GFP}_{2}$ tagged TDP-43 reporters, HeLa cells were grown for at least 2 passages in DMEM supplemented with $10 \%$ dialyzed FBS and were transiently transfected with the different $\mathrm{GCR}_{2}-\mathrm{GFP}_{2}-\mathrm{TDP}-43$ variants as described above. Import of the $\mathrm{GCR}_{2}-\mathrm{GFP}_{2}-$ TDP-43 reporters was induced by adding dexamethasone ( $5 \mu \mathrm{M}$ final concentration) in imaging medium (fluorobrite) and followed by live cell imaging using a spinning disk confocal microscope.

\section{$\underline{\text { SG association assay }}$}

HeLa cells were grown on high precision (No. 1.5) poly-L-lysine coated $12 \mathrm{~mm}$ coverslips, and after SG induction with MG132 (10 $\mu \mathrm{M}$ for 2.5-3h), cells were permeabilized for $2 \mathrm{x} 2 \mathrm{~min}$ with $0.004-0.005 \%$ digitonin in KPB (20 mM potassium phosphate $\mathrm{pH} 7.4,5 \mathrm{mM} \mathrm{Mg}(\mathrm{OAc})_{2}, 200$ $\mathrm{mM}$ KOAc, $1 \mathrm{mM}$ EGTA, $2 \mathrm{mM}$ DTT and $1 \mathrm{mg} / \mathrm{ml}$ each aprotinin, pepstatin and leupeptin). Soluble proteins were removed by several, stringent washes ( $4 \mathrm{x} 4 \mathrm{~min}$ in KPB on ice) before blocking nuclear pores by $15 \mathrm{~min}$ incubation with $200 \mathrm{mg} / \mathrm{ml}$ wheat germ agglutinin (WGA) on ice. Cells were then incubated for $30 \mathrm{~min}$ at RT with $100 \mathrm{nM}$ TDP-43-MBP-His6. For comparison of in vitro phosphorylated TDP-43 with controls, proteins were either subjected to the in vitro phosphorylation reaction or mock treated (Wt, 12D) in absence of kinase or ATP before exchanging the buffer to KPB using 40K Zeba spin desalting columns (Thermo). Subsequently, cells were washed ( $3 \mathrm{x} 5 \mathrm{~min}$ in KPB on ice) to remove unbound TDP-43-MBPHis 6 and processed by immunostaining to visualize SGs. SGs and TDP-43-MBP-His6 were visualized by G3BP1 immunostaining (rabbit anti G3BP1antibody, Proteintech, Cat.No: 13057-2-AP) and MBP immunostaining (by mouse anti MBP antibody, Proteintech, Cat.No: 66003-1-Ig), respectively. On Fig. 4B for clarity, signals were converted to grey values in the individual channels (upper two rows). In the merge (lower row), G3BP1 is shown in magenta, TDP-43-MBP-His6 in green, white pixels indicate colocalization. Nuclei were counterstained with DAPI (turquoise).

\section{siRNA-mediated knockdown of TDP-43}

TDP-43 knockdown was achieved using the pre-designed TARDBPHSS118765 siRNA (Invitrogen) as described in (Dormann et al., 2009). Briefly, $20 \mathrm{nM}$ siRNA was transfected into HeLa cells using RNAimax (Thermo) transfection reagent. Knockdown was analyzed $48 \mathrm{~h}$ post transfection by immunohistochemistry using mouse anti TDP-43 antibody (Proteintech, Cat.No: 60019-2-Ig) and immunoblotting using rabbit anti TDP-43 C-Term antibody (Proteintech, Cat.No: 12892-1-AP) to detect TDP-43 and mouse anti alpha-Tubulin antibody (Proteintech, Cat.No: 66031-1-Ig) for detection of $\alpha$-Tubulin as a control.

\section{RNA extraction and RT-PCR to analyze TDP-43 splice targets}

TDP-43 expression was silenced in HeLa cells by siRNA as described above and $24 \mathrm{~h}$ later cells were transfected with siRNA-resistant pcDNA5-FRT-TO-Myc-hTDP43 constructs (Wt, $12 \mathrm{D}$ and $12 \mathrm{~A}$ ). $48 \mathrm{~h}$ after transfection, cells were harvested and total RNA was extracted using an RNeasy mini kit from Qiagen. cDNA was synthesized using 500 ng of total RNA, M-MLV reverse transcriptase polymerase (Invitrogen), and $6 \mu \mathrm{M}$ of random hexamer primer (NEB). cDNA was amplified with Taq DNA polymerase (NEB) using the forward (FW) and reverse (RV) primers targeting the SKAR gene (FW - 5'CCTTCATAAACCCACCCATTGGGACAG3'; RV- 5'GTGGTGGAGAAAGCCGCCTGAG3') (Fiesel et al., 2012) and the BIM gene (FW- 
5'TCTGAGTGTGACCGAGAAGG3'; RV - 5'TCTTGGGCGATCCATATCTC 3') (Tollervey et al., 2011). PCR products were separated by electrophoresis on a $2.5 \%$ agarose gel containing GelRed (Sigma).

Electrophoretic-mobility shift assays (EMSA)

The TDP-43 autoregulatory RNA site (Ayala et al., 2011) located in the TARDBP 3'UTR (5'UCACAGGCCGCGUCUUUGACGGUGGGUGUCCCAUUUUUAUCCGCUACUCUUU AUUUCAUGGAGUCGUAUCAACGCUAUGAACGCAAGGCUGUGAUAUGGAACCAG AAGGCUGUCUGAACUUUUGAAACCUUGUGUGGGAUUGAUGGUGGUGCCGAGG CAUGAAAGGCUAGUAUGAGCGAGAAAAGGAGAGAGCGCGUGCAGAGACUUGG UGGUGCAUAAUGGAUAUUUUUUAACUUGGCGAGAUGUGUCUCUCAAUCCUGU GGCUUUGGUGAGAGAGUGUGCAGAGAGCAAUGAUAGCAAAUAAUGUACGAAU GUUUUUUGCAUUCAAAGGACAUCCACAUCUGUUGGAAGACUUUUAAGUGAGU UUUUGUUCUUAGAUAACCCACAUUAGAUGAAUGUGUUAAGUGAAAUGAUACU UGUACUCCCCCUACCCCUUUGUCAACUGCUGUG) was in vitro transcribed from double-stranded DNA templates and Cy5-labeled using the HyperScribe ${ }^{\text {TM }}$ T7 High Yield Cy5 RNA Labeling Kit (APExBIO, Cat.No: K1062) per manufacturer's instructions. (UG) 12 RNA (5' UGUGUGUGUGUGUGUGUGUGUGUG) was chemically synthesized with the addition of a 5' Cy5-label (Metabion). $16 \mathrm{nM}$ of Cy5-labeled RNA was mixed with varying amounts of TDP-43 Wt, 12A, and 12D $(0-1.6 \mu \mathrm{M})$. Binding reactions $(20 \mu \mathrm{L})$ were incubated in binding buffer [20 mM NaPO 4 (pH 8), $150 \mathrm{mM} \mathrm{NaCl}, 1 \mathrm{mM}$ DTT, $5 \mathrm{mM} \mathrm{MgCl} 2,0.5 \mathrm{mg} / \mathrm{mL} \mathrm{BSA}, 0.1$ $\mathrm{mg} / \mathrm{mL}$ yeast tRNA, 5\% glycerol, $1 \mathrm{U} / \mu \mathrm{L}$ RNase Inhibitor (NEB)] for 20 minutes at RT before loading onto a $1 \mathrm{~mm}$ thick non-denaturing polyacrylamide gel $(6 \%)$ in $0.5 \mathrm{x}$ TBE. Gels were run at $100 \mathrm{~V}$ for 1 hour at RT. Gels were imaged with a Typhoon ${ }^{\mathrm{TM}}$ FLA 9500 laser scanner.

\section{Immunostaining}

All steps were performed at RT. HeLa cells were fixed in 4\% formaldehyde in PBS for 10 min, permeabilized for 5 min using $0.2 \%(\mathrm{v} / \mathrm{v})$ Triton X-100 in PBS and subsequently blocked in blocking buffer ( $5 \%$ goat or donkey serum in $0.1 \%$ saponine in PBS) for $30 \mathrm{~min}$. Primary and secondary antibodies were diluted in blocking buffer and applied each for $1 \mathrm{~h}$ and washed three times using 0.1\% saponine in PBS. TDP-43 was stained using mouse anti TDP-43 antibody (Proteintech), SGs were stained using goat anti TIA1 antibody (Santa Cruz, Cat. No: sc-48371) or rabbit anti G3BP1 antibody (Proteintech) and DNA was stained with DAPI at $0.5 \mu \mathrm{g} / \mathrm{ml}$ in PBS for 5 min. Coverslips were then mounted on glass slides with ProLong ${ }^{\text {TM }}$ Diamond Antifade reagent (Life Technologies) and dried overnight at RT.

Hippocampal neurons cultured on glass coverslips were washed twice with PBS and fixed for 10 min at RT using 4\% paraformaldehyde and 4\% sucrose in PBS. Primary antibody as well as secondary antibody $(1: 400)$ were diluted in GDB buffer $(0.1 \%$ gelatin, $0.3 \%$ Triton X-100, $450 \mathrm{mM} \mathrm{NaCl}, 16 \mathrm{mM}$ sodium phosphate $\mathrm{pH}$ 7.4). Primary antibody (Mouse anti Map2, Sigma, Cat\# M1406, RRID: AB_477171) was incubated overnight at $4^{\circ} \mathrm{C}$ while secondary antibodies was applied for $1 \mathrm{~h}$ at $\mathrm{RT}$, each followed by three times washing with PBS. Coverslips were mounted using Vectashield Vibrance with DAPI (Biozol) to counterstain nuclei. 


\section{Bright and wide-field microscopy}

Imaging of unlabeled TDP-43 condensates was done by bright-field microscopy on an Axio Oberver.Z1 wide-field fluorescence microscope, using a 63x/1.40 Oil objective and an AxioCam 506 (Zeiss, Oberkochen, Germany).

\section{Confocal microscopy}

Confocal microscopy was performed using an inverted Leica SP8 microscope and the LAS X imaging program (Leica), provided by the Bioimaging core facility of the Biomedical Center, which included the excitation lasers for 405, 488, 552 and $638 \mathrm{~nm}$. Images were acquired using two-fold frame averaging with a $63 \times 1.4$ oil objective, with an image pixel size of $180 \mathrm{~nm}$ for A1.488-TDP-43 aggregates and fixed cells, and $59 \mathrm{~nm}$ for images of cells subjected to the SG association assay. The following fluorescence settings were used for detection: DAPI: 419-442 nm, GFP: 498-533 nm, Alexa 555: 562-598 nm, Alexa 647: 650-700 nm. Recording was performed sequentially using a conventional photomultiplier tube (PMT) to avoid bleedthrough.

\section{Spinning disc confocal microscopy}

a) Nuclear transport assay imaging:

Images were acquired for a duration of $50 \mathrm{~min}$ in $2.5 \mathrm{~min}$ intervals at $36.5^{\circ} \mathrm{C}$ and $5 \% \mathrm{CO}_{2}$ (EMBLEM environmental chamber) using an inverted microscope (Axio Observer.Z1; Carl Zeiss, Oberkochen, Germany) equipped with a confocal spinning disc (CSU-X1; Yokogawa, Tokyo, Japan) and a 63x/1.4 oil immersion lens. Images were acquired using the $488 \mathrm{~nm}$ SD laser line and an EM-CCD camera (EvolveDelta; Photomoetrics) at bin 1x1.

b) Fusion events and Fluorescence Recovery after Photobleaching (FRAP)

Experiments were performed on an inverted microscope (Axio Observer.Z1; Carl Zeiss, Oberkochen, Germany) equipped with a confocal spinning disk unit (CSU-X1; Yokogawa, Tokyo, Japan) and an oil immersion lens of 100x/1.46 Oil Ph3. Images recording the dynamics of TDP-43 condensates were obtained using a EM-CCD camera (EvolveDelta; Photomoetrics), with a bin $1 \mathrm{x} 1$ in a recording mode of $5 \mathrm{sec}$ intervals in a block of $3 \mathrm{~min}$. Images of TDP-43 condensates after bleaching were acquired with bin $1 \times 1$ in streaming mode for $1.5 \mathrm{sec}$ followed by a block of 2 min where images were recorded in intervals of $5 \mathrm{sec}$. Experiments were performed at $\mathrm{RT}$ and $\geq 11$ condensates were analyzed per condition in three independent experiments. Localized photobleaching ("half-bleach") was obtained using a laser scanning device (UGA-42 Geo; Rapp OptoElectronic, Hamburg, Germany). The "Geo" module allowed for simultaneous laser illumination within hardware-defined shapes of different sizes. For this experiment, an illumination size of $\sim 4 \mu \mathrm{m}$ in a square-like shape was used. The targeting structure was half bleached to approximately $70 \%$ of the initial intensity using a $473 \mathrm{~nm}$ diode laser (DL-473/75; Rapp OptoElectronic, Hamburg, Germany).

\section{Quantification and analysis}

\section{Droplet quantification}

Wide-field images of droplets were processed and quantified and measured using Image J/Fiji software. First, a bandpass filter of 20 pixels was applied to all images in order to reveal some details and thresholds were adjusted to optimally include all droplets. Finally, droplets were 
counted and measured by their size and roundness [ $4 *$ area/ $\left(\pi^{*}\right.$ major_axis $\left.{ }^{\wedge}\right)$, or the inverse of the aspect ratio] using the command Analyze Particles, excluding the detection of particles with a circularity below 0.3 and/or an area smaller than 3 pixels. Statistical analyses were performed in GraphPad Prism 8.

\section{Analysis of cellular images}

Analysis of the nuclear transport assay was performed using Image J/Fiji software by measuring loss in cytoplasmic fluorescence over time and normalizing $\mathrm{t}=0 \mathrm{~min}$ to 1 .

Images of cells from the SG association assay (Hutten and Dormann, 2020) were processed and analyzed using Image J/Fiji software, applying linear enhancement for brightness and contrast and implemented plugins for measurement of pixel intensities in SGs.

Quantification of Myc-hTDP-43 recruitment into SGs was performed using Image J/Fiji software. First, SGs from TDP-43-positive cells were selected using the Wand tracing tool and a band of $1 \mu \mathrm{m}$ representing a proxy for the cytosol was drawn around all selected SGs using the 'Make Band' command. Then, all pixel intensities for both SG and band selections was extracted for the TDP-43 channel. After subtraction of the background signal from all measured values, calculation of the SG/band ratio was performed for each SG and normalized SG/band ratio $=0$ to 1 .

Analysis of Myc-hTDP-43 recruitment into NBs was performed by counting the number of cells with positive TDP-43 nuclear condensates, excluding cells expressing TDP-43 staining only in the cytoplasm. Profile of TDP-43 nuclear staining was performed using Image J/Fiji software by using the 'Plot Profile' command, which quantifies the gray values along the indicated lines.

All statistical analyses were performed in GraphPad Prism 8.

\section{FRAP analysis}

FRAP analysis were performed using Image J/Fiji software by calculating the fluorescence intensity over time $(\mathrm{I}(\mathrm{t}))$ using the macro Time Series Analyzer command and the following formula:

$$
I(t)=[R O I 1(t)-R O I 3(t)] /[R O I 2(t)-R O I 3(t)]
$$

ROI1 corresponds to the averaged gray values of the bleached area, and ROI2 to the averaged gray values of the total droplet. ROI3 corresponds to the averaged background values. Values were further normalized to the initial fluorescence by dividing $I(t)$ by the mean gray value of the initial 1 time step before bleaching $\langle\mathrm{I}(1)>$. This way bleached areas were corrected for background noise and bleaching due to imaging over time. 


\section{References}

Abraham, M.J., Murtola, T., Schulz, R., Páll, S., Smith, J.C., Hess, B., and Lindahl, E. (2015). GROMACS: High performance molecular simulations through multi-level parallelism from laptops to supercomputers. SoftwareX 1-2, 19-25.

Afroz, T., Hock, E.M., Ernst, P., Foglieni, C., Jambeau, M., Gilhespy, L.A.B., Laferriere, F., Maniecka, Z., Pluckthun, A., Mittl, P., et al. (2017). Functional and dynamic polymerization of the ALS-linked protein TDP-43 antagonizes its pathologic aggregation. Nat Commun 8 , 45 .

Alberti, S. (2017). Phase separation in biology. Curr Biol 27, R1097-R1102.

Alberti, S., and Dormann, D. (2019). Liquid-Liquid Phase Separation in Disease. Annu Rev Genet 53, 171-194.

Alberti, S., and Hyman, A.A. (2021). Biomolecular condensates at the nexus of cellular stress, protein aggregation disease and ageing. Nat Rev Mol Cell Biol.

Alquezar, C., Arya, S., and Kao, A.W. (2020). Tau Post-translational Modifications: Dynamic Transformers of Tau Function, Degradation, and Aggregation. Front Neurol 11, 595532.

Avendano-Vazquez, S.E., Dhir, A., Bembich, S., Buratti, E., Proudfoot, N., and Baralle, F.E. (2012). Autoregulation of TDP-43 mRNA levels involves interplay between transcription, splicing, and alternative polyA site selection. Genes Dev 26, 1679-1684.

Ayala, Y.M., De Conti, L., Avendano-Vazquez, S.E., Dhir, A., Romano, M., D'Ambrogio, A., Tollervey, J., Ule, J., Baralle, M., Buratti, E., et al. (2011). TDP-43 regulates its mRNA levels through a negative feedback loop. EMBO J 30, 277-288.

Babinchak, W.M., Haider, R., Dumm, B.K., Sarkar, P., Surewicz, K., Choi, J.K., and Surewicz, W.K. (2019). The role of liquid-liquid phase separation in aggregation of the TDP43 low-complexity domain. J Biol Chem 294, 6306-6317.

Bah, A., and Forman-Kay, J.D. (2016). Modulation of Intrinsically Disordered Protein Function by Post-translational Modifications. J Biol Chem 291, 6696-6705.

Benayad, Z., von Bulow, S., Stelzl, L.S., and Hummer, G. (2021). Simulation of FUS Protein Condensates with an Adapted Coarse-Grained Model. J Chem Theory Comput 17, 525-537.

Bolognesi, B., Faure, A.J., Seuma, M., Schmiedel, J.M., Tartaglia, G.G., and Lehner, B. (2019). The mutational landscape of a prion-like domain. Nat Commun 10, 4162.

Brady, O.A., Meng, P., Zheng, Y., Mao, Y., and Hu, F. (2011). Regulation of TDP-43 aggregation by phosphorylation and p62/SQSTM1. J Neurochem 116, 248-259.

Buratti, E. (2015). Functional Significance of TDP-43 Mutations in Disease. Adv Genet 91, 153.

Buratti, E. (2018). TDP-43 post-translational modifications in health and disease. Expert Opin Ther Targets 22, 279-293.

Bussi, G., Donadio, D., and Parrinello, M. (2007). Canonical sampling through velocity rescaling. J Chem Phys 126, 014101.

Ceballos, A.V., McDonald, C.J., and Elbaum-Garfinkle, S. (2018). Methods and Strategies to Quantify Phase Separation of Disordered Proteins. Methods Enzymol 611, 31-50.

Choksi, D.K., Roy, B., Chatterjee, S., Yusuff, T., Bakhoum, M.F., Sengupta, U., Ambegaokar, S., Kayed, R., and Jackson, G.R. (2014). TDP-43 Phosphorylation by casein 
kinase Iepsilon promotes oligomerization and enhances toxicity in vivo. Hum Mol Genet 23, 1025-1035.

Conicella, A.E., Zerze, G.H., Mittal, J., and Fawzi, N.L. (2016). ALS Mutations Disrupt Phase Separation Mediated by alpha-Helical Structure in the TDP-43 Low-Complexity CTerminal Domain. Structure 24, 1537-1549.

Dormann, D., Capell, A., Carlson, A.M., Shankaran, S.S., Rodde, R., Neumann, M., Kremmer, E., Matsuwaki, T., Yamanouchi, K., Nishihara, M., et al. (2009). Proteolytic processing of TAR DNA binding protein-43 by caspases produces C-terminal fragments with disease defining properties independent of progranulin. J Neurochem.

Ederle, H., Funk, C., Abou-Ajram, C., Hutten, S., Funk, E.B.E., Kehlenbach, R.H., Bailer, S.M., and Dormann, D. (2018). Nuclear egress of TDP-43 and FUS occurs independently of Exportin-1/CRM1. Sci Rep 8, 7084.

Fiesel, F.C., Weber, S.S., Supper, J., Zell, A., and Kahle, P.J. (2012). TDP-43 regulates global translational yield by splicing of exon junction complex component SKAR. Nucleic Acids Res 40, 2668-2682.

French, R.L., Grese, Z.R., Aligireddy, H., Dhavale, D.D., Reeb, A.N., Kedia, N., Kotzbauer, P.T., Bieschke, J., and Ayala, Y.M. (2019). Detection of TAR DNA-binding protein 43 (TDP43) oligomers as initial intermediate species during aggregate formation. J Biol Chem 294, 6696-6709.

Gowers, R.J., Linke, M., Barnoud, J., Reddy, T.J.E., Melo, M.N., Seyler, S.L., Domański, J., Dotson, D.L., Buchoux, S., Kenney, I.M., et al. (2016). MDAnalysis: A Python Package for the Rapid Analysis of Molecular Dynamics Simulations. Proceedings of the 15th Python in Science Conference.

Guo, Q., Lehmer, C., Martinez-Sanchez, A., Rudack, T., Beck, F., Hartmann, H., PerezBerlanga, M., Frottin, F., Hipp, M.S., Hartl, F.U., et al. (2018). In Situ Structure of Neuronal C9orf72 Poly-GA Aggregates Reveals Proteasome Recruitment. Cell 172, 696-705 e612.

Haj-Yahya, M., Gopinath, P., Rajasekhar, K., Mirbaha, H., Diamond, M.I., and Lashuel, H.A. (2020). Site-Specific Hyperphosphorylation Inhibits, Rather than Promotes, Tau Fibrillization, Seeding Capacity, and Its Microtubule Binding. Angew Chem Int Ed Engl 59, 4059-4067.

Halfmann, R., and Lindquist, S. (2008). Screening for amyloid aggregation by SemiDenaturing Detergent-Agarose Gel Electrophoresis. J Vis Exp.

Hasegawa, M., Arai, T., Nonaka, T., Kametani, F., Yoshida, M., Hashizume, Y., Beach, T.G., Buratti, E., Baralle, F., Morita, M., et al. (2008). Phosphorylated TDP-43 in frontotemporal lobar degeneration and amyotrophic lateral sclerosis. Ann Neurol 64, 60-70.

Hipp, M.S., Kasturi, P., and Hartl, F.U. (2019). The proteostasis network and its decline in ageing. Nat Rev Mol Cell Biol 20, 421-435.

Hofweber, M., and Dormann, D. (2019). Friend or foe-Post-translational modifications as regulators of phase separation and RNP granule dynamics. J Biol Chem 294, 7137-7150.

Hutten, S., and Dormann, D. (2020). A Quantitative Assay to Measure Stress Granule Association of Proteins and Peptides in Semi-permeabilized Human Cells. Bio-protocol 10, e3846.

Hutten, S., Usluer, S., Bourgeois, B., Simonetti, F., Odeh, H.M., Fare, C.M., Czuppa, M., Hruska-Plochan, M., Hofweber, M., Polymenidou, M., et al. (2020). Nuclear Import 
Receptors Directly Bind to Arginine-Rich Dipeptide Repeat Proteins and Suppress Their Pathological Interactions. Cell Rep 33, 108538.

Inukai, Y., Nonaka, T., Arai, T., Yoshida, M., Hashizume, Y., Beach, T.G., Buratti, E., Baralle, F.E., Akiyama, H., Hisanaga, S., et al. (2008). Abnormal phosphorylation of Ser409/410 of TDP-43 in FTLD-U and ALS. FEBS Lett 582, 2899-2904.

Josephs, K.A., Whitwell, J.L., Weigand, S.D., Murray, M.E., Tosakulwong, N., Liesinger, A.M., Petrucelli, L., Senjem, M.L., Knopman, D.S., Boeve, B.F., et al. (2014). TDP-43 is a key player in the clinical features associated with Alzheimer's disease. Acta Neuropathol 127, 811-824.

Kametani, F., Nonaka, T., Suzuki, T., Arai, T., Dohmae, N., Akiyama, H., and Hasegawa, M. (2009). Identification of casein kinase-1 phosphorylation sites on TDP-43. Biochem Biophys Res Commun 382, 405-409.

Kametani, F., Obi, T., Shishido, T., Akatsu, H., Murayama, S., Saito, Y., Yoshida, M., and Hasegawa, M. (2016). Mass spectrometric analysis of accumulated TDP-43 in amyotrophic lateral sclerosis brains. Sci Rep 6, 23281.

Lancaster, A.K., Nutter-Upham, A., Lindquist, S., and King, O.D. (2014). PLAAC: a web and command-line application to identify proteins with prion-like amino acid composition.

Bioinformatics 30, 2501-2502.

Larsen, A.H., Wang, Y., Bottaro, S., Grudinin, S., Arleth, L., and Lindorff-Larsen, K. (2020). Combining molecular dynamics simulations with small-angle X-ray and neutron scattering data to study multi-domain proteins in solution. PLoS Comput Biol 16, e1007870.

Li, H.R., Chiang, W.C., Chou, P.C., Wang, W.J., and Huang, J.R. (2018). TAR DNA-binding protein 43 (TDP-43) liquid-liquid phase separation is mediated by just a few aromatic residues. J Biol Chem 293, 6090-6098.

Li, H.Y., Yeh, P.A., Chiu, H.C., Tang, C.Y., and Tu, B.P. (2011). Hyperphosphorylation as a defense mechanism to reduce TDP-43 aggregation. PLoS One 6, e23075.

Li, Q., Babinchak, W.M., and Surewicz, W.K. (2021). Cryo-EM structure of amyloid fibrils formed by the entire low complexity domain of TDP-43. Nat Commun 12, 1620.

Liachko, N.F., McMillan, P.J., Guthrie, C.R., Bird, T.D., Leverenz, J.B., and Kraemer, B.C. (2013). CDC7 inhibition blocks pathological TDP-43 phosphorylation and neurodegeneration. Ann Neurol 74, 39-52.

Liachko, N.F., McMillan, P.J., Strovas, T.J., Loomis, E., Greenup, L., Murrell, J.R., Ghetti, B., Raskind, M.A., Montine, T.J., Bird, T.D., et al. (2014). The tau tubulin kinases TTBK1/2 promote accumulation of pathological TDP-43. PLoS Genet 10, e1004803.

Ling, S.C., Polymenidou, M., and Cleveland, D.W. (2013). Converging mechanisms in ALS and FTD: disrupted RNA and protein homeostasis. Neuron 79, 416-438.

Mackenzie, I.R., Rademakers, R., and Neumann, M. (2010). TDP-43 and FUS in amyotrophic lateral sclerosis and frontotemporal dementia. Lancet Neurol 9, 995-1007.

Marrink, S.J., Risselada, H.J., Yefimov, S., Tieleman, D.P., and de Vries, A.H. (2007). The MARTINI force field: coarse grained model for biomolecular simulations. J Phys Chem B $111,7812-7824$.

Martin, E.W., Holehouse, A.S., Peran, I., Farag, M., Incicco, J.J., Bremer, A., Grace, C.R., Soranno, A., Pappu, R.V., and Mittag, T. (2020). Valence and patterning of aromatic residues determine the phase behavior of prion-like domains. Science 367, 694-699. 
Martin, E.W., Thomasen, F.E., Milkovic, N.M., Cuneo, M.J., Grace, C.R., Nourse, A., Lindorff-Larsen, K., and Mittag, T. (2021). Interplay of folded domains and the disordered low-complexity domain in mediating hnRNPA1 phase separation. Nucleic Acids Res 49, 2931-2945.

Martinez-Gonzalez, L., Rodriguez-Cueto, C., Cabezudo, D., Bartolome, F., Andres-Benito, P., Ferrer, I., Gil, C., Martin-Requero, A., Fernandez-Ruiz, J., Martinez, A., et al. (2020). Motor neuron preservation and decrease of in vivo TDP-43 phosphorylation by protein CK1delta kinase inhibitor treatment. Sci Rep 10, 4449.

May, S., Hornburg, D., Schludi, M.H., Arzberger, T., Rentzsch, K., Schwenk, B.M., Grasser, F.A., Mori, K., Kremmer, E., Banzhaf-Strathmann, J., et al. (2014). C9orf72 FTLD/ALSassociated Gly-Ala dipeptide repeat proteins cause neuronal toxicity and Unc119 sequestration. Acta Neuropathol 128, 485-503.

McGibbon, R.T., Beauchamp, K.A., Harrigan, M.P., Klein, C., Swails, J.M., Hernandez, C.X., Schwantes, C.R., Wang, L.P., Lane, T.J., and Pande, V.S. (2015). MDTraj: A Modern Open Library for the Analysis of Molecular Dynamics Trajectories. Biophys J 109, 15281532.

Michaud-Agrawal, N., Denning, E.J., Woolf, T.B., and Beckstein, O. (2011). MDAnalysis: a toolkit for the analysis of molecular dynamics simulations. J Comput Chem 32, 2319-2327.

Molliex, A., Temirov, J., Lee, J., Coughlin, M., Kanagaraj, A.P., Kim, H.J., Mittag, T., and Taylor, J.P. (2015). Phase separation by low complexity domains promotes stress granule assembly and drives pathological fibrillization. Cell 163, 123-133.

Monticelli, L., Kandasamy, S.K., Periole, X., Larson, R.G., Tieleman, D.P., and Marrink, S.J. (2008). The MARTINI Coarse-Grained Force Field: Extension to Proteins. J Chem Theory Comput 4, 819-834.

Morris, M., Knudsen, G.M., Maeda, S., Trinidad, J.C., Ioanoviciu, A., Burlingame, A.L., and Mucke, L. (2015). Tau post-translational modifications in wild-type and human amyloid precursor protein transgenic mice. Nat Neurosci 18, 1183-1189.

Nedelsky, N.B., and Taylor, J.P. (2019). Bridging biophysics and neurology: aberrant phase transitions in neurodegenerative disease. Nat Rev Neurol 15, 272-286.

Neumann, M., Kwong, L.K., Lee, E.B., Kremmer, E., Flatley, A., Xu, Y., Forman, M.S., Troost, D., Kretzschmar, H.A., Trojanowski, J.Q., et al. (2009). Phosphorylation of S409/410 of TDP-43 is a consistent feature in all sporadic and familial forms of TDP-43 proteinopathies. Acta Neuropathol 117(2), 137-149.

Neumann, M., Sampathu, D.M., Kwong, L.K., Truax, A.C., Micsenyi, M.C., Chou, T.T., Bruce, J., Schuck, T., Grossman, M., Clark, C.M., et al. (2006). Ubiquitinated TDP-43 in frontotemporal lobar degeneration and amyotrophic lateral sclerosis. Science 314, 130-133.

Nonaka, T., Suzuki, G., Tanaka, Y., Kametani, F., Hirai, S., Okado, H., Miyashita, T., Saitoe, M., Akiyama, H., Masai, H., et al. (2016). Phosphorylation of TAR DNA-binding Protein of $43 \mathrm{kDa}$ (TDP-43) by Truncated Casein Kinase 1delta Triggers Mislocalization and Accumulation of TDP-43. J Biol Chem 291, 5473-5483.

Parrinello, M., and Rahman, A. (1981). Polymorphic Transitions in Single Crystals: A New Molecular Dynamics Method. J Appl Phys 52, 7182 - 7190.

Patel, A., Lee, H.O., Jawerth, L., Maharana, S., Jahnel, M., Hein, M.Y., Stoynov, S., Mahamid, J., Saha, S., Franzmann, T.M., et al. (2015). A Liquid-to-Solid Phase Transition of the ALS Protein FUS Accelerated by Disease Mutation. Cell 162, 1066-1077. 
Piana, S., Donchev, A.G., Robustelli, P., and Shaw, D.E. (2015). Water dispersion interactions strongly influence simulated structural properties of disordered protein states. $\mathrm{J}$ Phys Chem B 119, 5113-5123.

Pietrek, L.M., Stelzl, L.S., and Hummer, G. (2020). Hierarchical Ensembles of Intrinsically Disordered Proteins at Atomic Resolution in Molecular Dynamics Simulations. J Chem Theory Comput 16, 725-737.

Ratti, A., and Buratti, E. (2016). Physiological functions and pathobiology of TDP-43 and FUS/TLS proteins. J Neurochem 138 Suppl 1, 95-111.

Robustelli, P., Piana, S., and Shaw, D.E. (2018). Developing a molecular dynamics force field for both folded and disordered protein states. Proc Natl Acad Sci U S A 115, E4758-E4766.

Salado, I.G., Redondo, M., Bello, M.L., Perez, C., Liachko, N.F., Kraemer, B.C., Miguel, L., Lecourtois, M., Gil, C., Martinez, A., et al. (2014). Protein kinase CK-1 inhibitors as new potential drugs for amyotrophic lateral sclerosis. J Med Chem 57, 2755-2772.

Taylor, L.M., McMillan, P.J., Liachko, N.F., Strovas, T.J., Ghetti, B., Bird, T.D., Keene, C.D., and Kraemer, B.C. (2018). Pathological phosphorylation of tau and TDP-43 by TTBK1 and TTBK2 drives neurodegeneration. Mol Neurodegener 13, 7.

Tollervey, J.R., Curk, T., Rogelj, B., Briese, M., Cereda, M., Kayikci, M., Konig, J., Hortobagyi, T., Nishimura, A.L., Zupunski, V., et al. (2011). Characterizing the RNA targets and position-dependent splicing regulation by TDP-43. Nat Neurosci 14, 452-458.

Tziortzouda, P., Van Den Bosch, L., and Hirth, F. (2021). Triad of TDP43 control in neurodegeneration: autoregulation, localization and aggregation. Nat Rev Neurosci 22, 197 208.

Wang, A., Conicella, A.E., Schmidt, H.B., Martin, E.W., Rhoads, S.N., Reeb, A.N., Nourse, A., Ramirez Montero, D., Ryan, V.H., Rohatgi, R., et al. (2018). A single N-terminal phosphomimic disrupts TDP-43 polymerization, phase separation, and RNA splicing. EMBO J 37 .

Wang, C., Duan, Y., Duan, G., Wang, Q., Zhang, K., Deng, X., Qian, B., Gu, J., Ma, Z., Zhang, S., et al. (2020). Stress Induces Dynamic, Cytotoxicity-Antagonizing TDP-43 Nuclear Bodies via Paraspeckle LncRNA NEAT1-Mediated Liquid-Liquid Phase Separation. Mol Cell 79, 443-458 e447.

Yerbury, J.J., Farrawell, N.E., and McAlary, L. (2020). Proteome Homeostasis Dysfunction: A Unifying Principle in ALS Pathogenesis. Trends Neurosci 43, 274-284. 


\section{Figures}

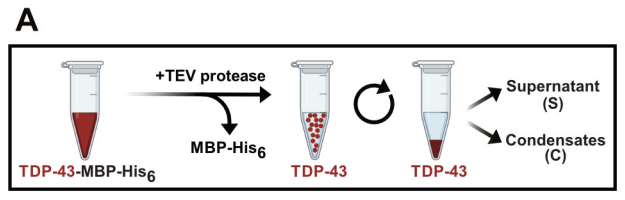

D
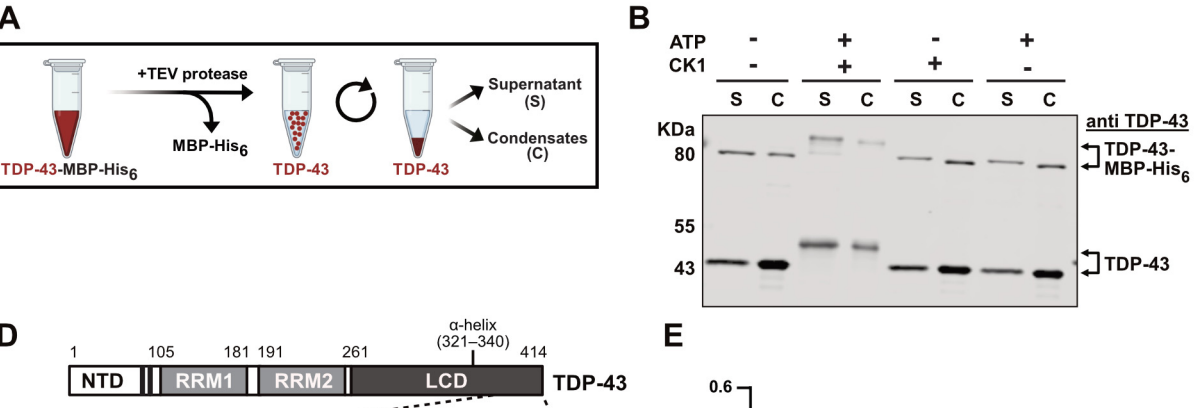

E

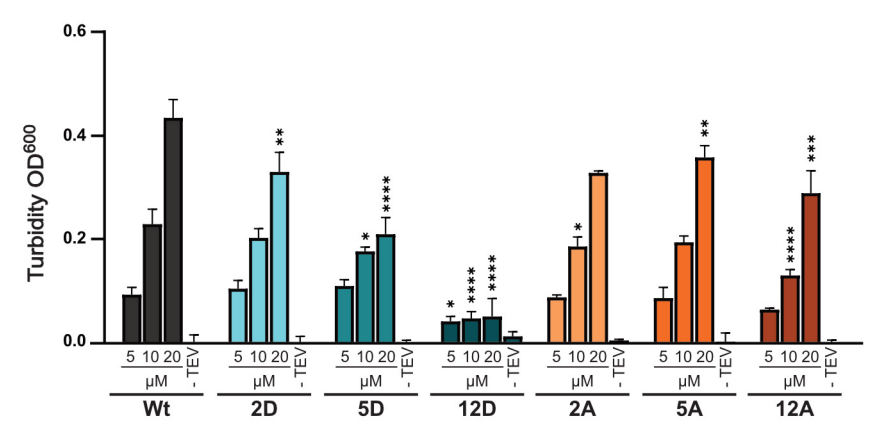

G

$\mathbf{F}$

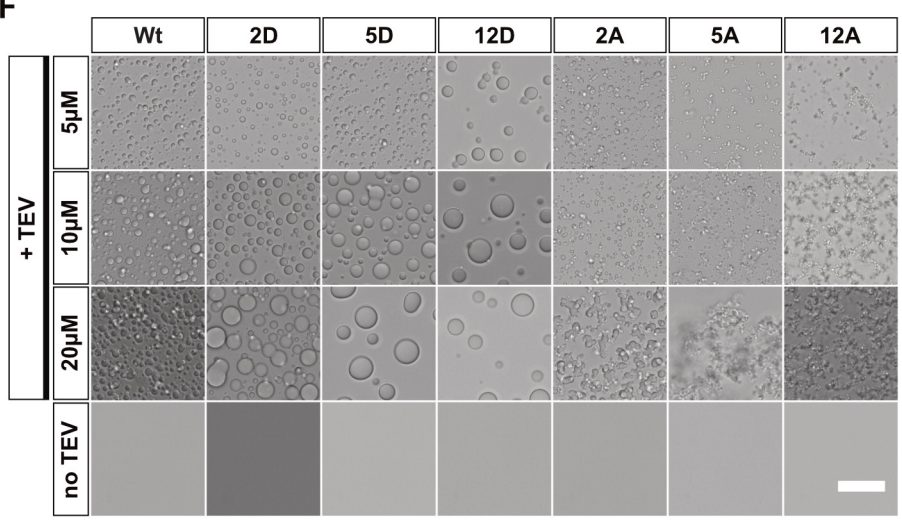

C

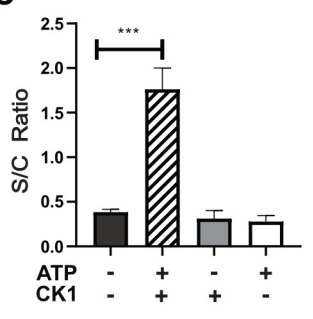

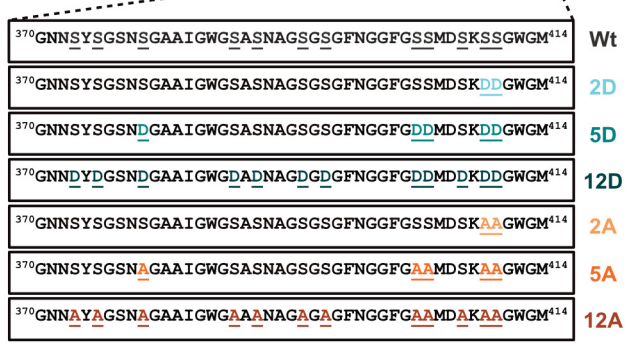
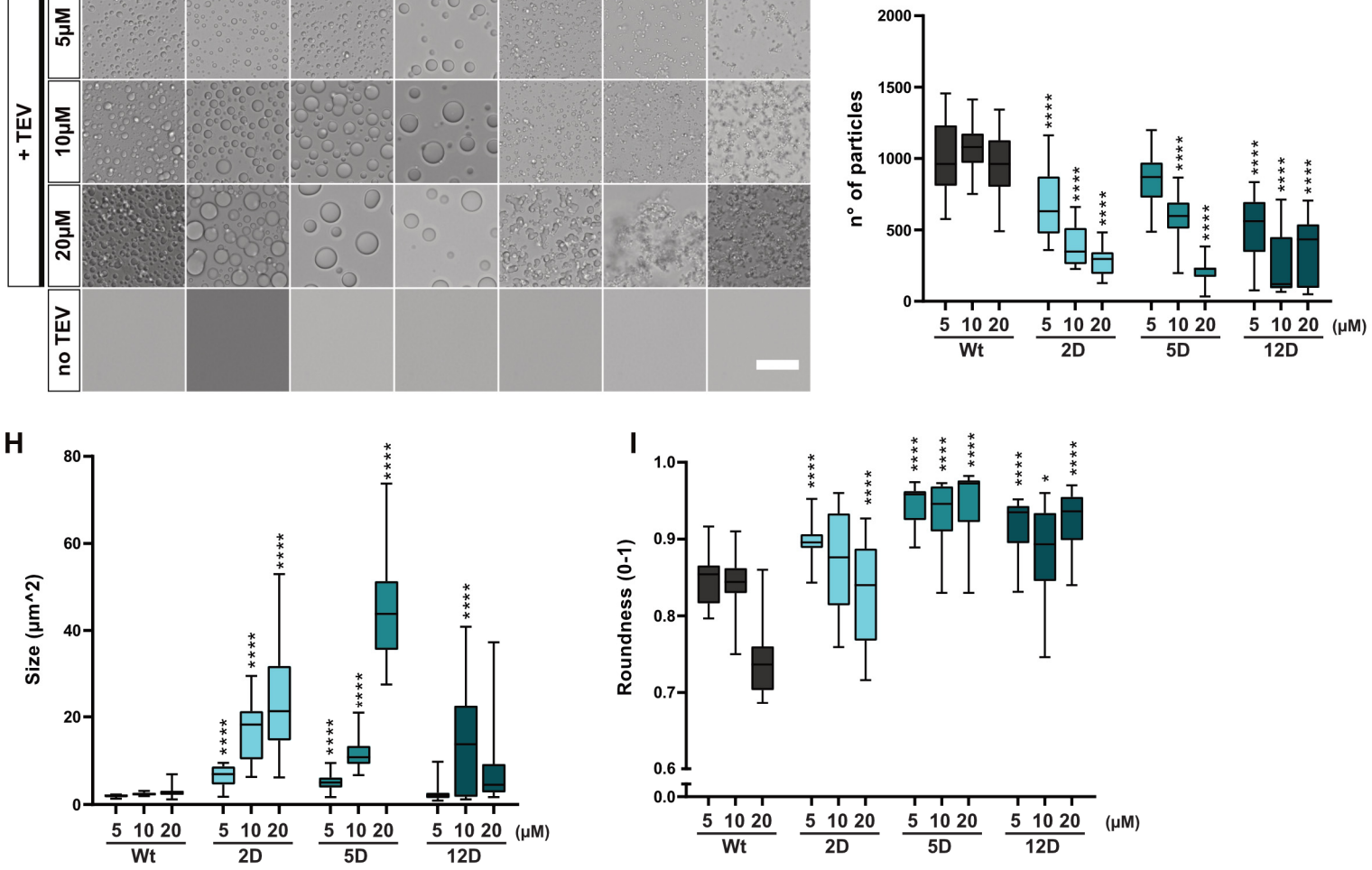

Figure 1. TDP-43 phosphorylation by CK1ס and C-terminal phosphomimetic substitutions reduce TDP-43 condensation in vitro.

A Scheme of sedimentation assay (created in BioRender.com): Phase separation of TDP-43 was induced by TEV protease cleavage, and condensates were pelleted by centrifugation.

B Sedimentation assay to quantify condensation of unmodified TDP-43 versus in vitro phosphorylated TDP-43 (+CK1 $\delta,+$ ATP) and controls (CK1 $\delta$ or ATP only); TDP-43 detected by Western blot (rabbit anti-TDP-43 N-term). 
C Quantification of band intensities shown as means of Supernatant/Condensate (S/C) ratio $(n=3) \pm$ SEM.

D Schematic diagram of TDP-43 and sequence of C-terminal region for $\mathrm{Wt}$, phosphomimetic (S-to-D) variants and control (S-to-A) variants.

E Turbidity measurements to quantify phase separation of the indicated TDP-43 variants at three different concentrations. Values represent means $(n=3) \pm$ SD.

F-I Representative bright field microscopic images of TDP-43 condensates, Bar, $25 \mu \mathrm{m}(\mathrm{F})$ and quantification of condensate number $(\mathrm{G})$, size $(\mathrm{H})$ and roundness $(\mathrm{I})$. Values represent means of all fields of view (FOV) from Min to Max (whiskers) of two replicates ( $\geq 22$ FOV per condition).

${ }^{*} \mathrm{p}<0.0332, * * \mathrm{p}<0.0021, * * * \mathrm{p}<0.0002$ and $* * * * \mathrm{p}<0.0001$ by one-way ANOVA with Dunnett's multiple comparison test to Wt (in C, E, G, H and I, respectively). 


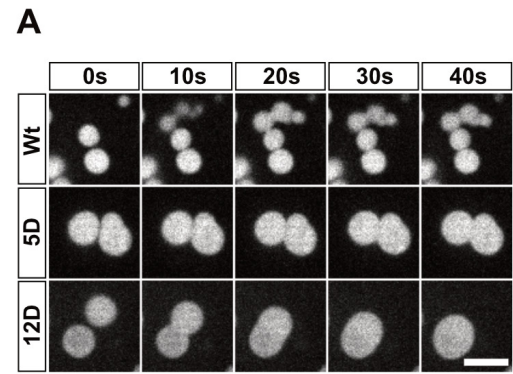

D

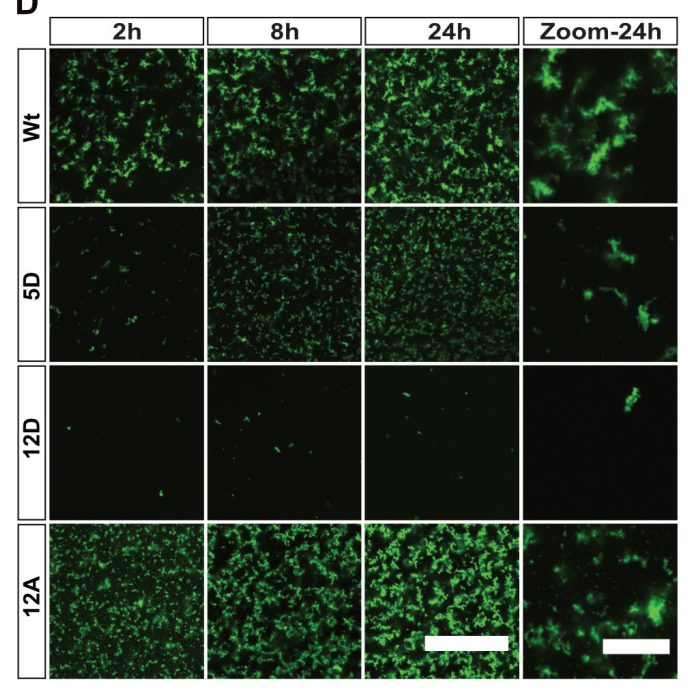

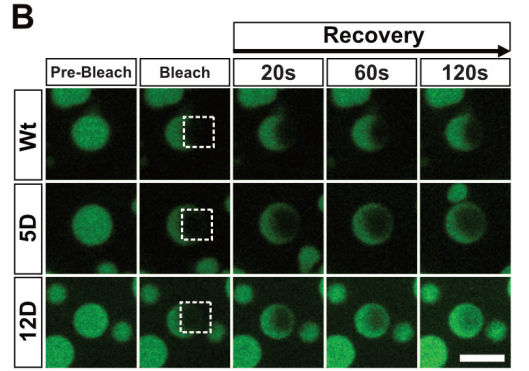

E
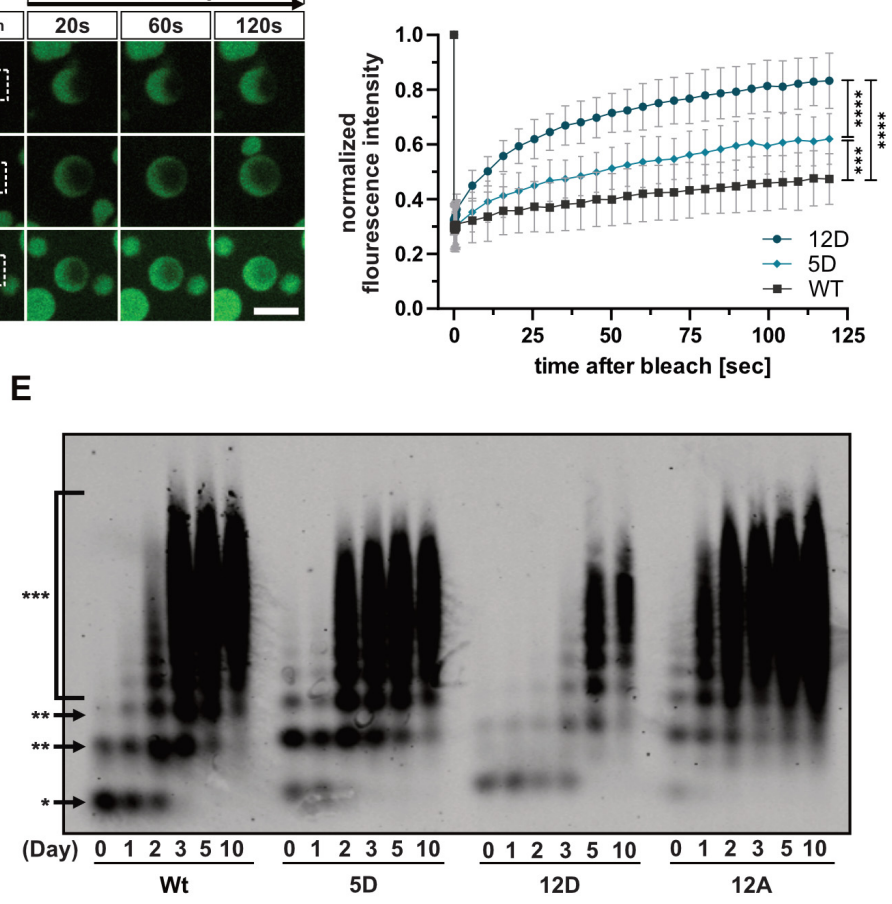

$\mathbf{F}$

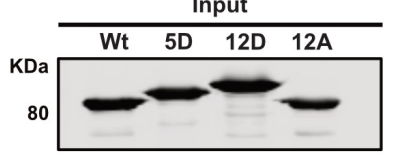

Figure 2. C-terminal phosphomimetic substitutions enhance liquidity of TDP-43 condensates and reduce TDP-43 aggregation in vitro.

A Representative still images of Alexa488-labeled TDP-43 condensates by spinning disc timelapse confocal microscopy. Wt condensates do not fuse, 5D condensates fuse slowly and 12D condensates readily fuse upon contact and relax into spherical droplets. Bar, $5 \mu \mathrm{m}$.

B Representative images of FRAP experiments at indicated time-points. Boxes indicate bleached area. Bar, $5 \mu \mathrm{m}$.

C FRAP curves after half-bleach of freshly formed Alexa488-labeled TDP-43 condensates. Values represent means $\pm \mathrm{SD}(\mathrm{n}=3)$ of $\geq 9$ droplets analyzed per condition. $* * * \mathrm{p}<0.0002$ and $* * * * p<0.0001$ by one-way ANOVA with Tukey's multiple comparison test for AUC of individual droplets.

D Confocal images of Alexa488-labeled TDP-43 aggregates formed in an in vitro aggregation assay (with TEV protease cleavage). Bar, $100 \mu \mathrm{m}$. Zoom shows magnified view of aggregates at the $24 \mathrm{~h}$ time point. Bar, $20 \mu \mathrm{m}$.

E SDD-AGE followed by TDP-43 Western blot to visualize SDS-resistant oligomers / high molecular weight species of TDP-43-MBP-His 6 in an in vitro aggregation assay (without TEV protease cleavage). Asterisks represent monomeric $(*)$, oligomeric $(* *)$ and polymeric $(* * *)$ species.

$\mathrm{F}$ Input of TDP-43 variants used in the SDD-AGE assay, detected by Western blot (anti-TDP$43 \mathrm{~N}$-term). 
A
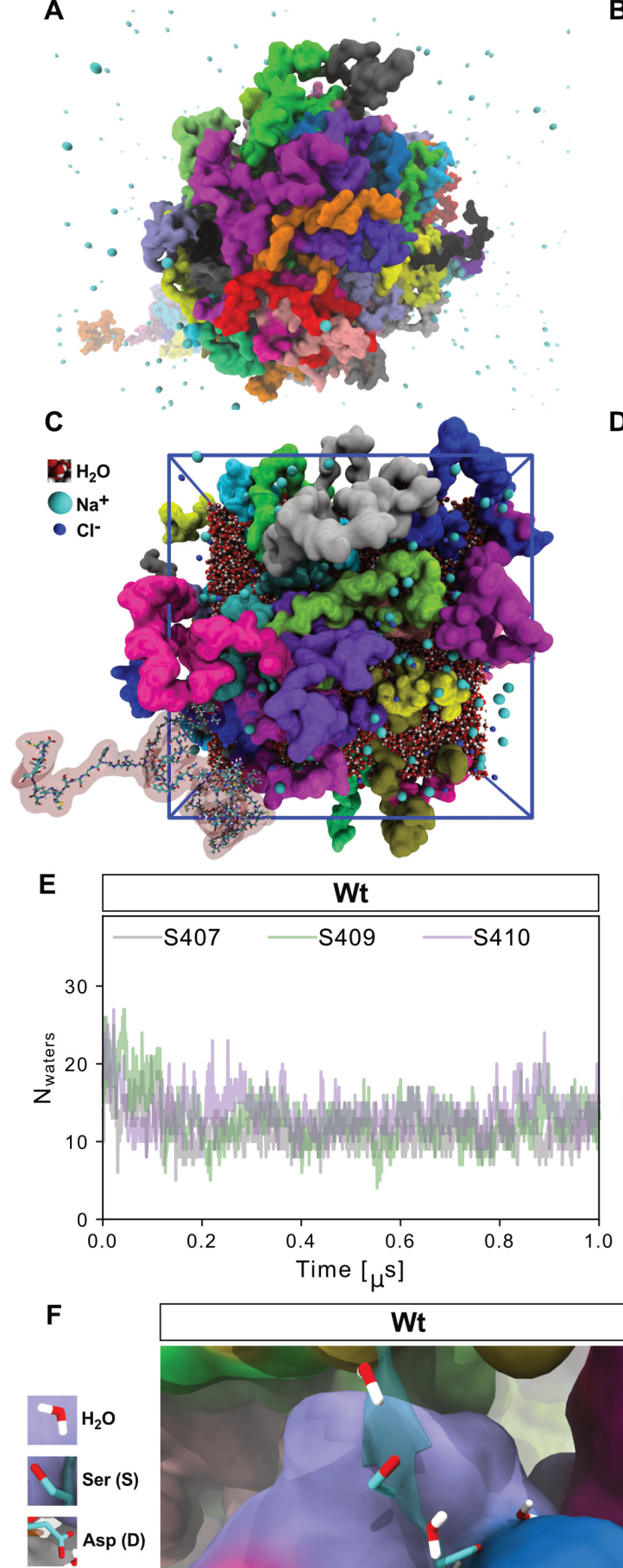

B

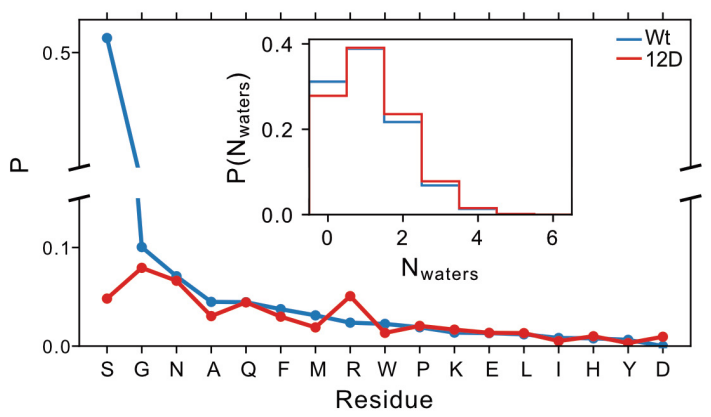

D
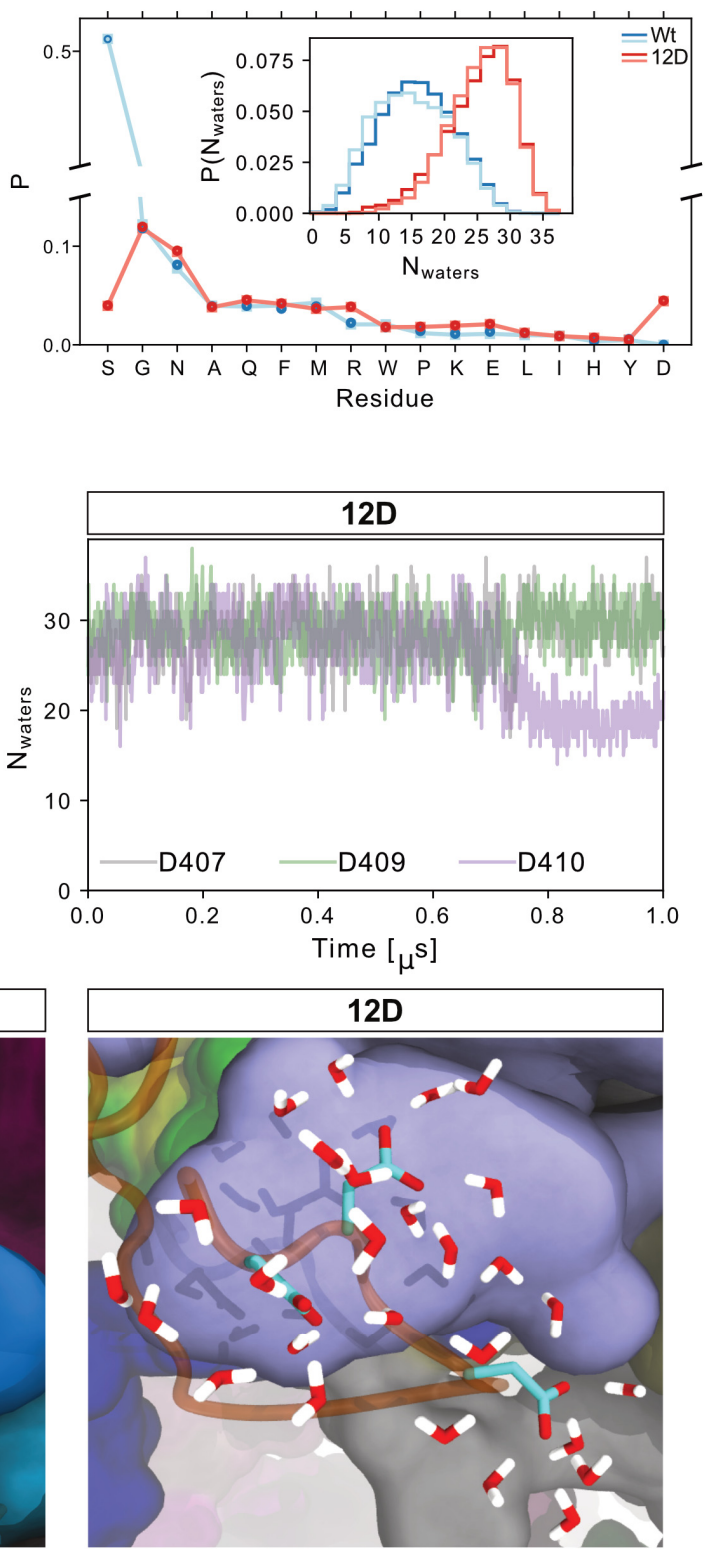

Figure 3: Atomistic and coarse-grained simulations of TDP-43 LCD: Phosphomimicking residues form fewer protein-protein interactions and more protein-solvent interactions.

A TDP-43 LCD phase separates in coarse-grained simulations with explicit solvent. Water omitted for clarity. Ions shown in cyan. Condensate of TDP-43 Wt LCD is shown, protein colored according to chain identity. 
B Normalized probability of protein-protein contacts by phosphomimicking aspartates in 12D and serines in Wt resolved by amino acid type from coarse-grained simulations. Error bars smaller than symbols. Inset: Distributions of the number of water molecules within $5 \AA$ of sidechains of phosphomimicking aspartates of $12 \mathrm{D}$ and corresponding serines in Wt in $3 \times 5 \mu \mathrm{s}$ segments from coarse-grained simulations. Data from different segments are on top of each other.

C Atomistic simulation setup of 32 TDP-43 LCDs. Different LCD chains shown in different colors in space-filling representation. For one chain (lower left), all atoms are shown to highlight the high-resolution atomistic description.

D Normalized probability of protein-protein contacts by phosphomimicking aspartates in 12D and serines in Wt resolved by amino acid type from atomistic simulations. Two $1 \mu$ s simulations are distinguished by color intensity. Inset: Distributions of the number of water molecules within $5 \AA$ of the sidechains of phosphomimicking aspartates of $12 \mathrm{D}$ and the corresponding serines in $\mathrm{Wt}$ from atomistic simulations.

E Time series of number of water molecules bound to Wt S407, S409, and S410 (chain 23) and 12D D407, D409, and D410 (chain 32) in atomistic molecular dynamics.

F Representative snapshots of atomistic simulations showing water within $3 \AA$ of Wt S407, S409, S410 with nearby LCDs in surface representation and 12D D407, D409, and D410. 
A

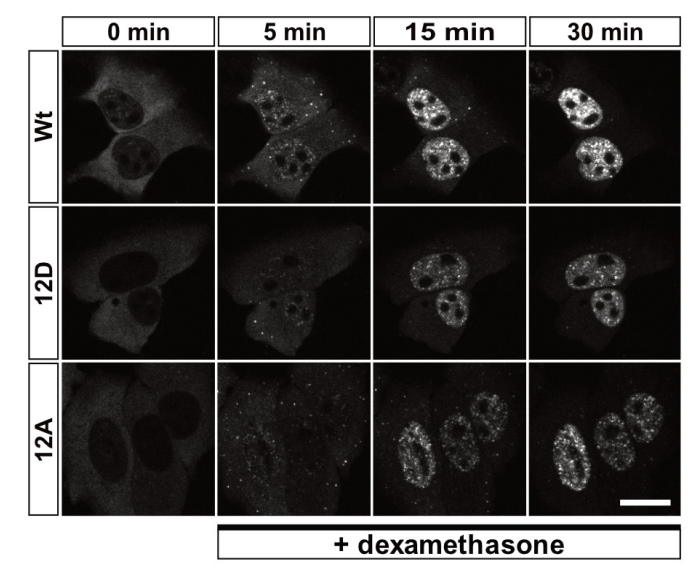

C

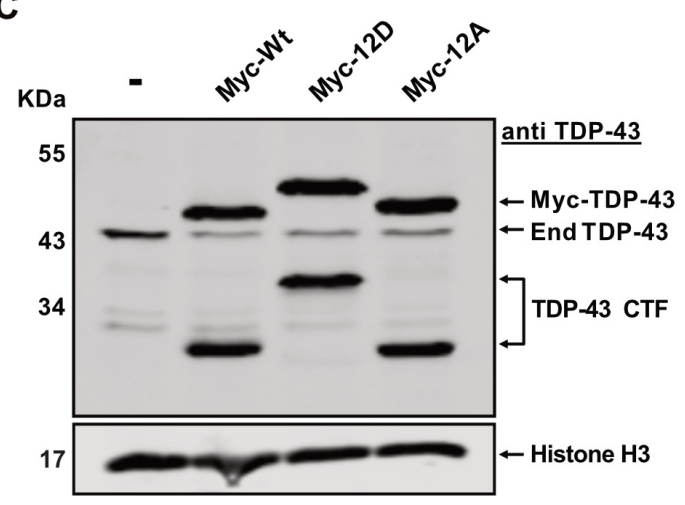

B
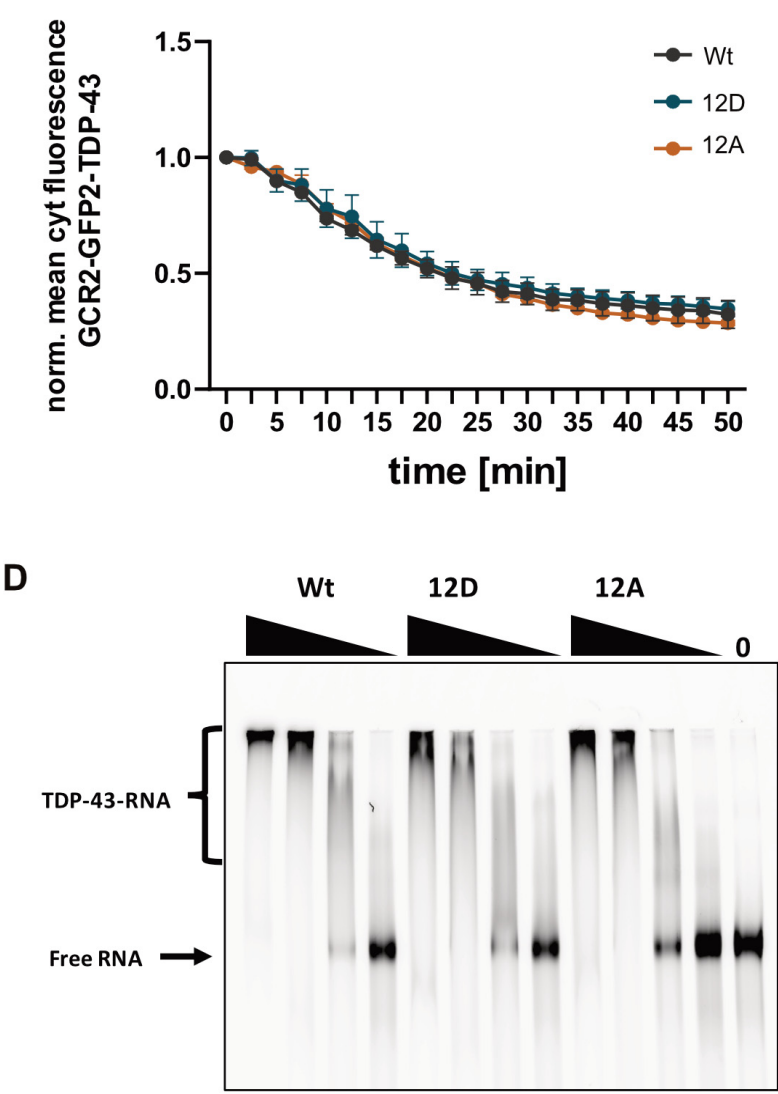

E

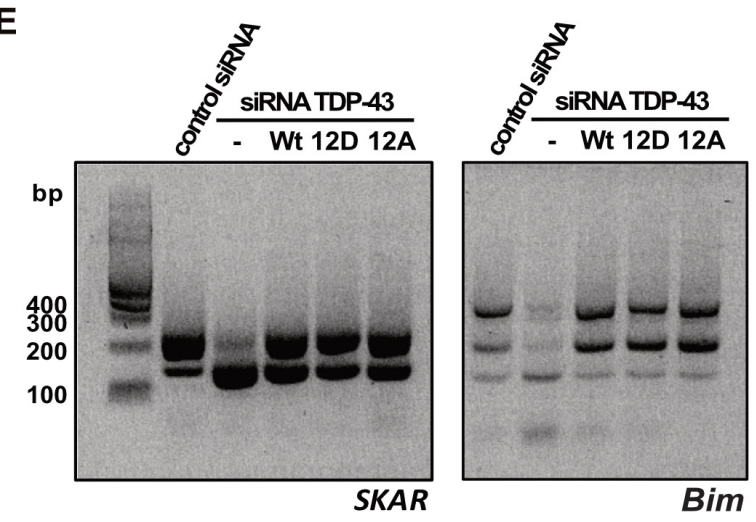

Figure 4. Phosphomimetic substitutions do not alter the rate of TDP-43 nuclear import and do not impair TDP-43 autoregulation, RNA-binding or alternative splicing function.

A Representative still images of $\mathrm{GCR}_{2}-\mathrm{EGFP}_{2}-\mathrm{TDP}-43 \mathrm{Wt}, 12 \mathrm{D}$ and $12 \mathrm{~A}$ before and during import triggered by addition of dexamethasone. Images were live recorded by spinning disc confocal microscopy. Bar, $20 \mu \mathrm{m}$.

B Quantification of the hormone-inducible nuclear import measured during a total time course of $50 \mathrm{~min}$. Values represent the mean fluorescence intensity of TDP-43-MBP-His 6 in the cytoplasm for three independent replicates \pm SEM ( $\geq 42$ cells per condition).

C Phosphomimetic 12D TDP-43 is competent in autoregulating TDP-43 expression. SDSPAGE followed by TDP-43 Western blot showing downregulation of endogenous TDP-43 through autoregulation (60) after $48 \mathrm{~h}$ expression of $\mathrm{Wt}, 12 \mathrm{D}$ and $12 \mathrm{~A}$ variants in HeLa cells. 
TDP-43 was detected using rabbit anti-TDP-43 C-term antibody (Proteintech), Histone H3 (rabbit anti-Histone H3 antibody, Abcam) was visualized as a loading control.

D Electrophoretic mobility shift assays (EMSA) of TDP-43-MBP-His6 variants (Wt, 12D and 12A) in a complex with TDP-43 autoregulatory RNA site (60). All TDP-43 variants form TDP43-RNA complexes equally well.

E Splicing analysis by RT-PCR of known TDP-43 splice targets (SKAR exon 3 and Bim exon 3 ) in HeLa cells. Silencing of endogenous TDP-43 by siRNA leads to altered splice isoforms of SKAR and Bim (second vs. first lane). These splicing alterations can be rescued by reexpression of TDP-43 Wt, but also $12 \mathrm{D}$ or $12 \mathrm{~A}$ variants, demonstrating that phosphomimetic TDP-43 is fully competent in regulation splicing of these TDP-43 splice targets. 
A

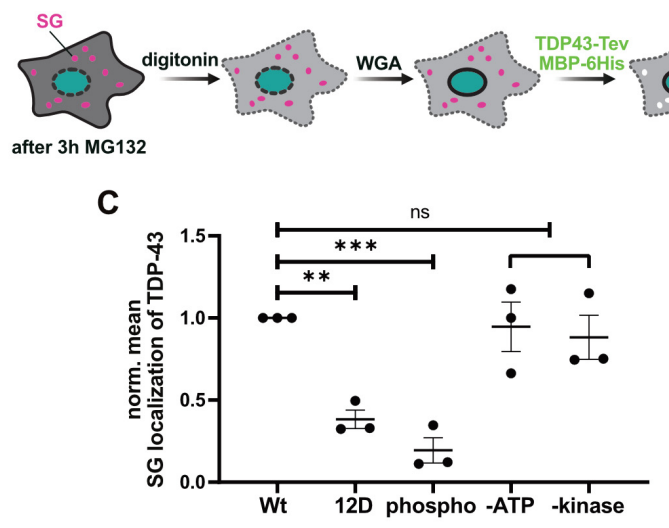

D

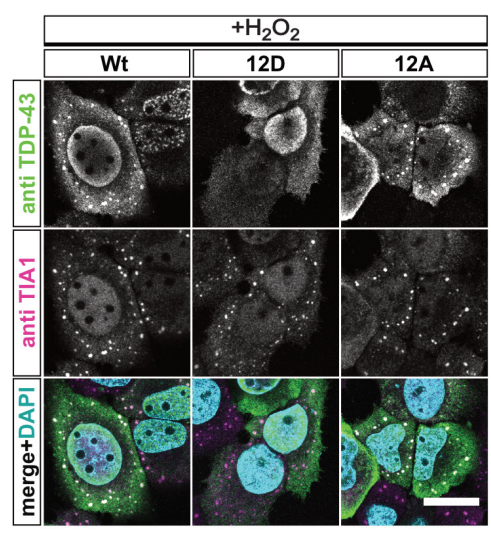

H

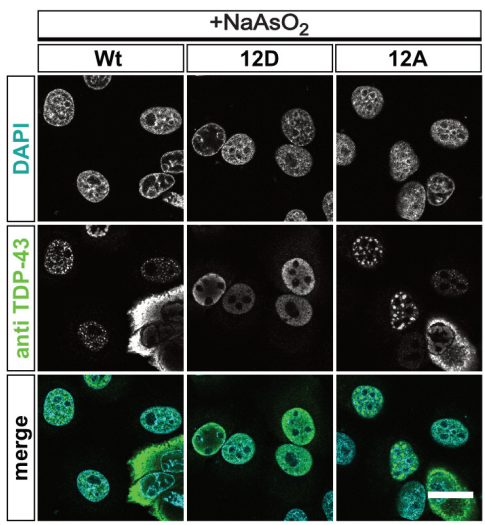

$\mathbf{E}$

G
B
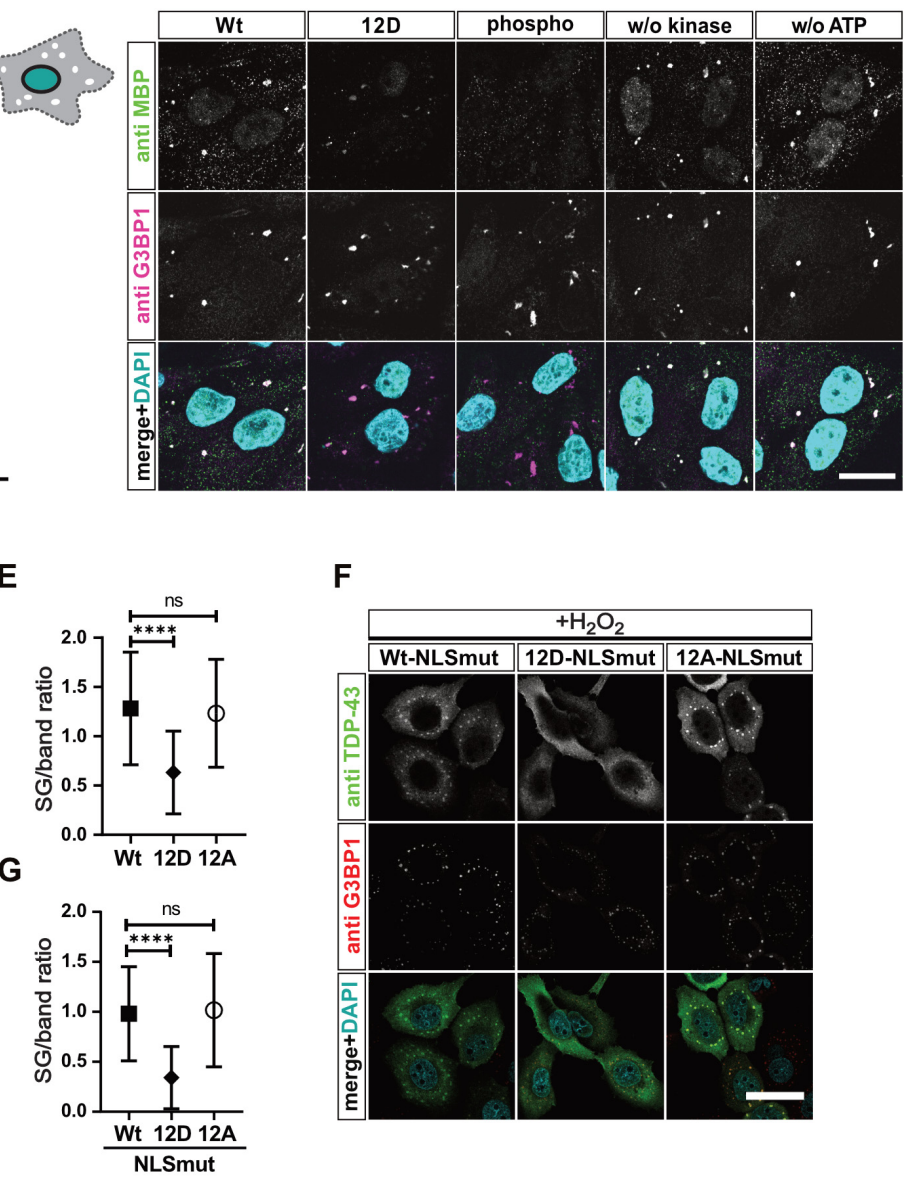

$\mathbf{F}$

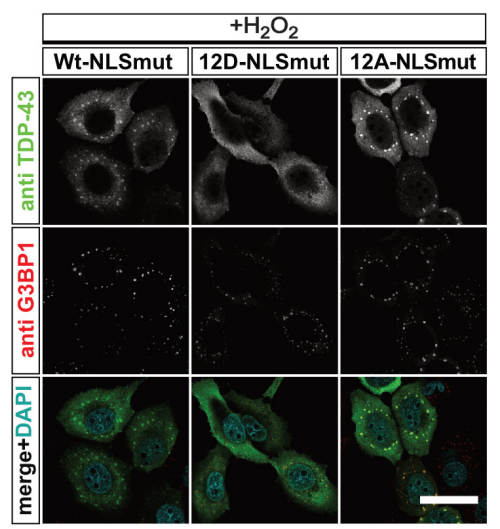

J

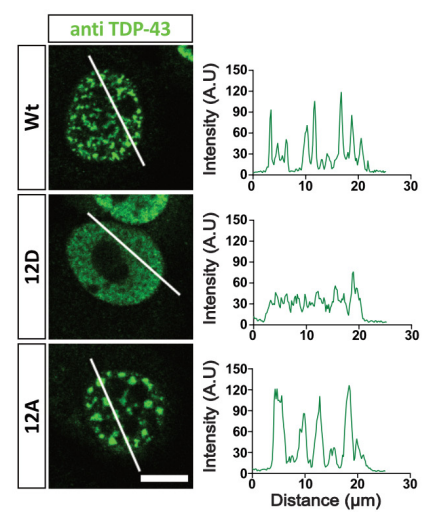

Figure 5. Phosphorylation and phosphomimetic substitutions reduce recruitment of TDP43 into stress-induced membrane-less organelles.

A Scheme of stress granule (SG) recruitment assay in semi-permeabilized cells.

B Reduced SG association of TDP-43 by $12 \mathrm{D}$ mutations or in vitro phosphorylation. Bar, 20 $\mu \mathrm{m}$.

C Quantification of TDP-43-MBP-His6 mean fluorescence intensity in SGs normalized to Wt $\pm \operatorname{SEM}(\mathrm{n}=3)(\geq 10$ cells; $\geq 46$ SGs each).

D SG recruitment of TDP-43 variants in intact HeLa cells in absence of endogenous TDP-43. After TDP-43 silencing and expression of NLSmut Wt, 12D and 12A variants, SGs were induced by $\mathrm{H}_{2} \mathrm{O}_{2}$ treatment and SG recruitment of TDP- 43 was monitored by TDP-43 and TIA1 immunostaining. For clarity, signals were converted to grey values in the individual channels 
(upper two rows). In the merge (lower row), nuclei were stained in DAPI (turquoise), TDP-43 (green) and TIA-1 (magenta). Bar, $25 \mu \mathrm{m}$.

E Quantification of TDP-43 in SGs versus cytoplasm \pm SD ( $n=2)$ ( $\geq 62$ cells; $\geq 234$ SGs each). F SG recruitment of different TDP-43-NLSmut variants in intact HeLa cells in the absence of endogenous TDP-43. After TDP-43 silencing and expression of NLSmut Wt, 12D and 12A variants, SGs were induced by $\mathrm{H}_{2} \mathrm{O}_{2}$ treatment and $\mathrm{SG}$ recruitment of TDP-43 was monitored by TDP-43 and G3BP1 immunostaining. For clarity, signals were converted to grey values in the individual channels (upper two rows). In the merge (lower row), nuclei were stained in DAPI (turquoise), TDP-43 (green) and G3BP1 (red). Bar, $40 \mu \mathrm{m}$.

G Quantification of TDP-43-NLS mutants in SGs versus band around SGs of two independent replicates $\pm \mathrm{SD}$. $* * * * \mathrm{p}<0.0001$ by one-way ANOVA with Dunnett's multiple comparison test to $\mathrm{Wt}$ ( $\geq 56$ cells; $\geq 315$ SGs per condition).

$\mathrm{H}$ Recruitment of TDP-43 into arsenite-induced nuclear bodies (NBs). Bar, $20 \mu \mathrm{m}$.

I Percentage of cells with TDP-43 in NBs \pm SD ( $n=3)$.

$\mathrm{J}$ Intensity profiles (right) of nuclear TDP-43 Wt, 12D and 12A variants (green) along white lines (left). Bar, $10 \mu \mathrm{m}$. 
A

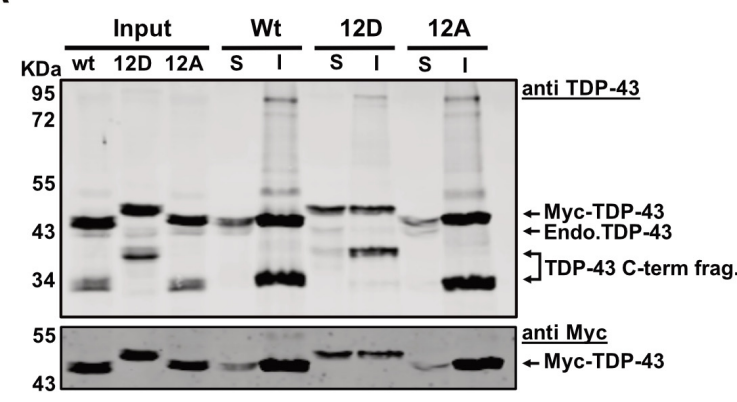

B

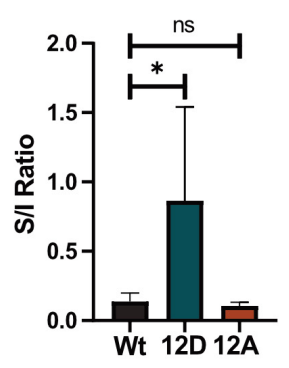

C

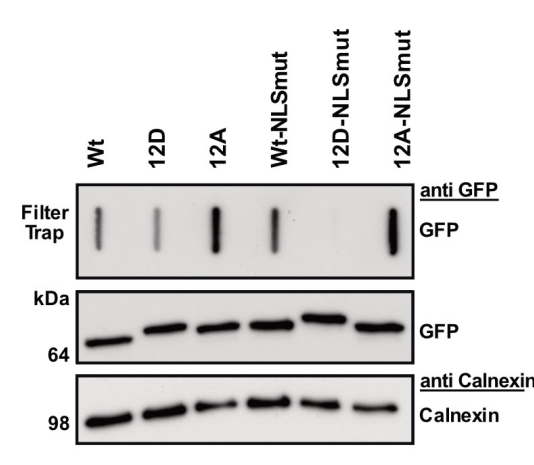

D

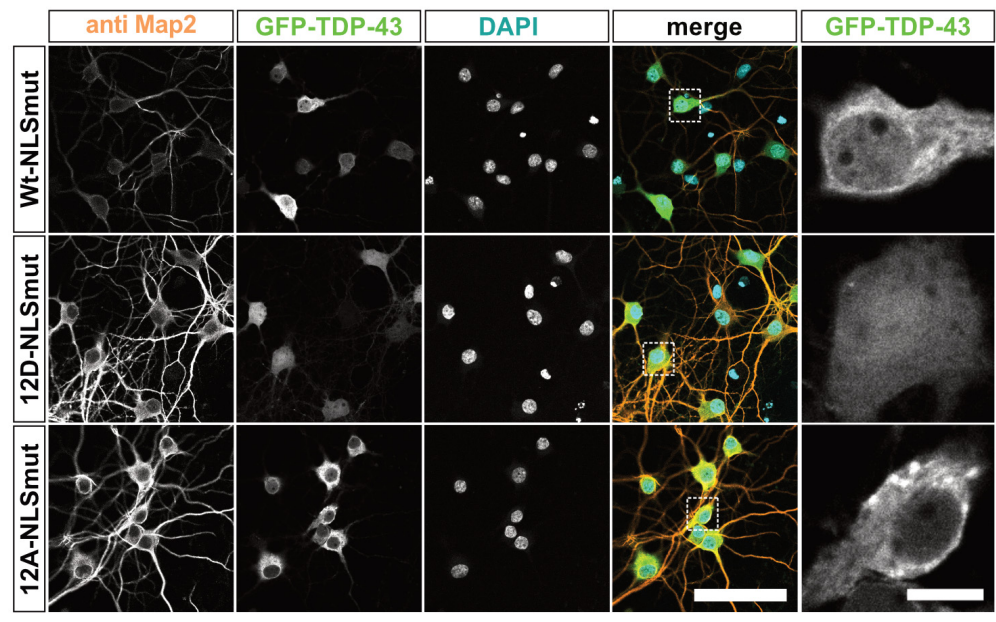

Figure 6. Phosphomimetic substitutions enhance TDP-43 solubility in HeLa cells and primary neurons.

A Biochemical fractionation into RIPA-soluble (S) and RIPA-insoluble (I) fractions to analyze solubility of the different TDP-43 variants (Wt, 12D and 12A) expressed in HeLa cells for 48 h. TDP-43 was detected by TDP-43 Western blot (upper blot, rabbit anti-TDP-43 C-term, Proteintech) and Myc Western blot (mouse anti-Myc 9E10).

B Quantification of TDP-43 variants (Wt, 12D and 12A) in (S) versus (I) fractions of 4 independent replicates $\pm \mathrm{SD}$. ${ }^{*} \mathrm{p}<0.0332$ by one-way ANOVA with Dunnett's multiple comparison test to $\mathrm{Wt}$.

C RIPA-insoluble material for TDP-43 variants ( \pm NLS mutation) in primary cortical neurons analyzed by filter-trap assay.

D Primary hippocampal neurons expressing TDP-43 Wt, 12D or 12A with additional NLS mutation. Bar, $80 \mu \mathrm{m}$. Right: Zoomed images of white squares (TDP-43 signal). Bar, $10 \mu \mathrm{m}$. $* * \mathrm{p}<0.0021, * * * \mathrm{p}<0.0002$ and $* * * * \mathrm{p}<0.0001$ by one-way ANOVA with Dunnett's multiple comparison test to Wt (in C, E and G). 


\section{Supplementary Figures}

A

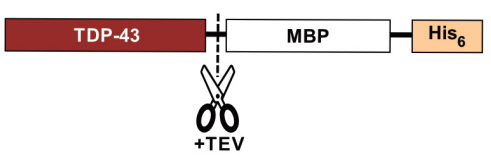

C

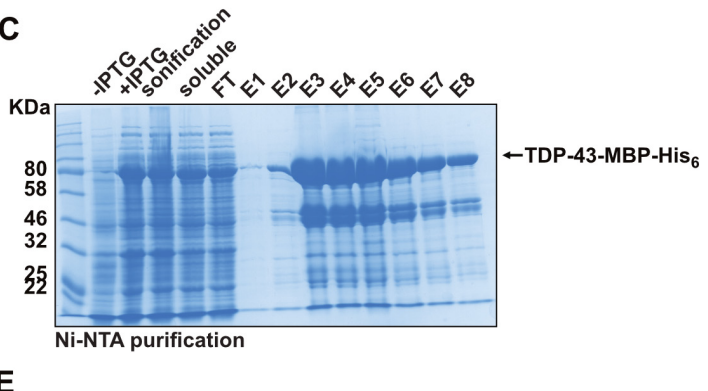

E

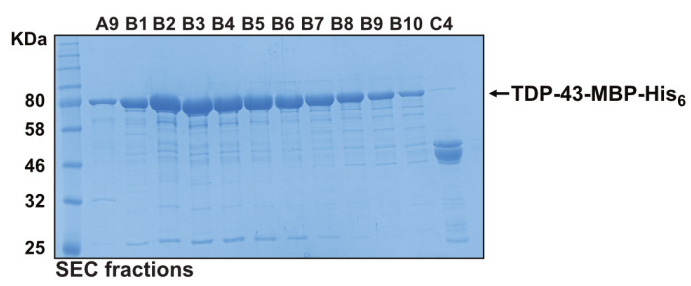

B

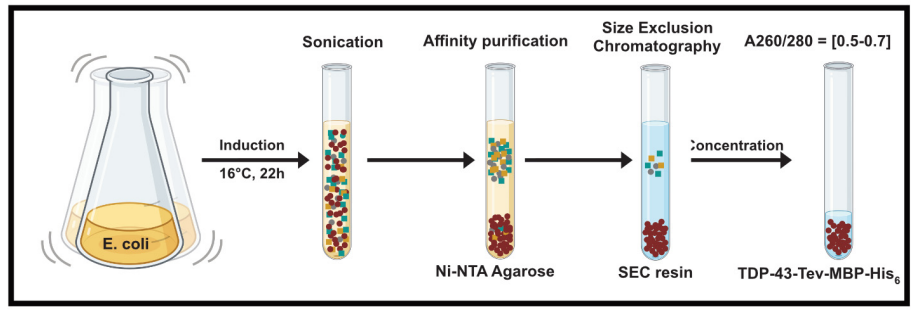

D

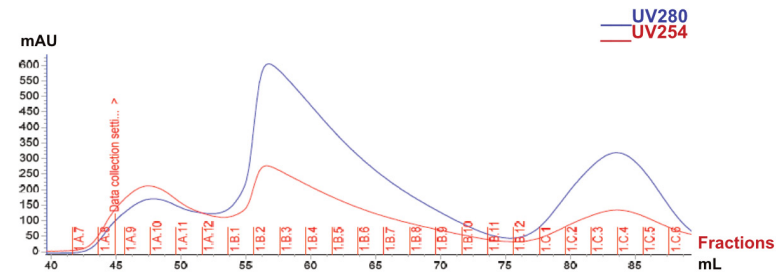

Fig. S1. TDP-43-MBP-His 6 purification.

A Schematic representation of purified full-length TDP-43 containing a solubilizing maltose binding protein (MBP) tag, a His6-tag and a TEV protease recognition site before the MBP tag.

B Schematic diagram of TDP-43-MBP-His6 expression and purification (created in BioRender.com). First, the construct is expressed in E. coli for $22 \mathrm{~h}$ at $16{ }^{\circ} \mathrm{C}$ and bacteria are lysed by sonication. Finally, the protein is purified under high salt conditions via Ni-NTA affinity purification and size exclusion chromatography (SEC) to obtain clean soluble TDP-43MBP-His6 (red circles), which is largely devoid of nucleic acids, as judged from the A260/280 ratio.

C Representative SDS-PAGE gel of different steps from protein expression to Ni-NTA affinity purification. First and second lines correspond to samples before (-IPTG) and after (+IPTG) induction of TDP-43-MBP-His6 expression. Third, fourth and fifth line correspond to samples after sonication, supernatant collection after spin down (soluble) and flow through (FT) after first wash with lysis buffer. The last 8 lines correspond to the consecutive elution steps (E1-E8) from the Ni-NTA agarose beads, which were pooled (E2-E6) and used for SEC. $\sim 89 \mathrm{kDa}$ bands were detected for TDP-43-MBP-His6 using Coomassie staining.

D Representative SEC profile showing the amount of protein (UV280) and nucleic acids (UV254) on the $y$-axis versus the volume $(\mathrm{mL})$ of the different fractions after SEC on the $\mathrm{x}-$ axis.

E Coomassie-stained SDS-PAGE gel showing the indicated fractions (A9-C4) of the SEC run displayed in (D). A9, B1-B10 and C4 are fraction samples from peaks A, B and C in the SEC 
profile (D) that represent TDP-43-MBP-His6 oligomeric, monomeric and cleaved species, respectively.

B

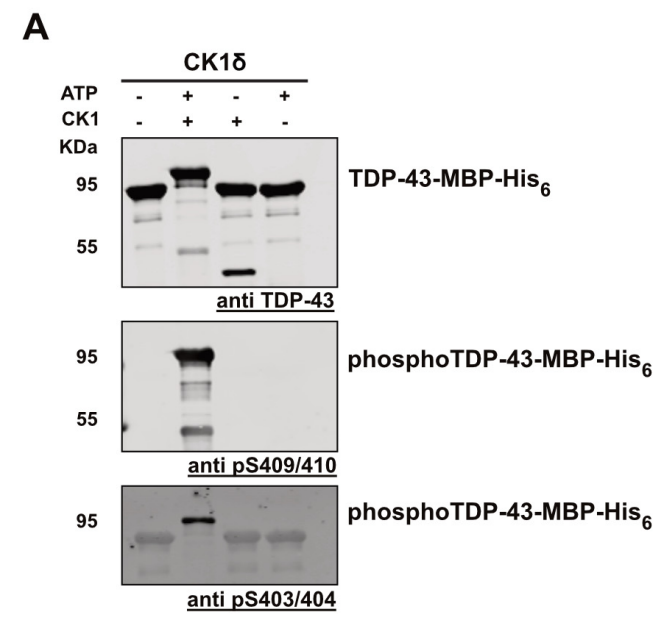

Trypsin digestion

MSEY IRVTEDENDEPIEIPSEDDGTVLLSTVTAQFPGACGLRYRNPVSQCMRGVRLVEGI LHAPDAGWGNLVYVVNYPKDNKRKMDETDAS SAVKVKRAVQKT SDLIVLGLPWKTTEQDL KEYFSTFGEVLMVQVKKDLKTGHSKGFGFVRFTEYETQVKVMSQRHMIDGRWCDCKLPNS KQSQDEPLRSRKVFVGRCTEDMTEDELREFF SQYGDVMDVFIPKPFRAFAFVTFADDQIA QSLCGEDLIIKGISVHISNAEPKHNSNRQLERSGRFGGNPGGFGNQGGFGNSRGGGAGLG NNQGSNMGGGMNF GAFSINPAMMAAAQAALQSSWGMMGMLASQQNQSGPSGNNQNQGNMQ REPNQAFGSGNNSYSGSNSGAAIGWGSASNAGSGSGFNGGFGSSMDSKSSGWGM

Fig. S2. Identification of TDP-43-MBP-His6 phospho-sites after in vitro phosphorylation with CK1ס.

A Identification of TDP-43 phospho-sites on in vitro phosphorylated TDP-43 (+CK1 $1 \delta,+\mathrm{ATP})$ in comparison to controls (-CK $1 \delta$-ATP; CK $1 \delta$ only; ATP only) by Western blot. Samples were run on SDS-PAGE and analyzed by Western blot using a rabbit anti-TDP-43 N-term antibody (Proteintech) to detect total TDP-43, rat anti-TDP-43-phospho Ser409/410 (clone 1D3, Helmholtz Center Munich) and mouse anti-TDP-43-phospho Ser403/404 (Proteintech, Cat.No: 66079-1-Ig) antibodies.

B Schematic diagrams showing sequence coverage in mass spectrometry after trypsin digest (underlined) and phosphorylated serine/threonine residues (orange) of in vitro phosphorylated TDP-43-MBP-His 6 with CK1 $\delta$ + ATP (one out of two representative experiments is shown). 

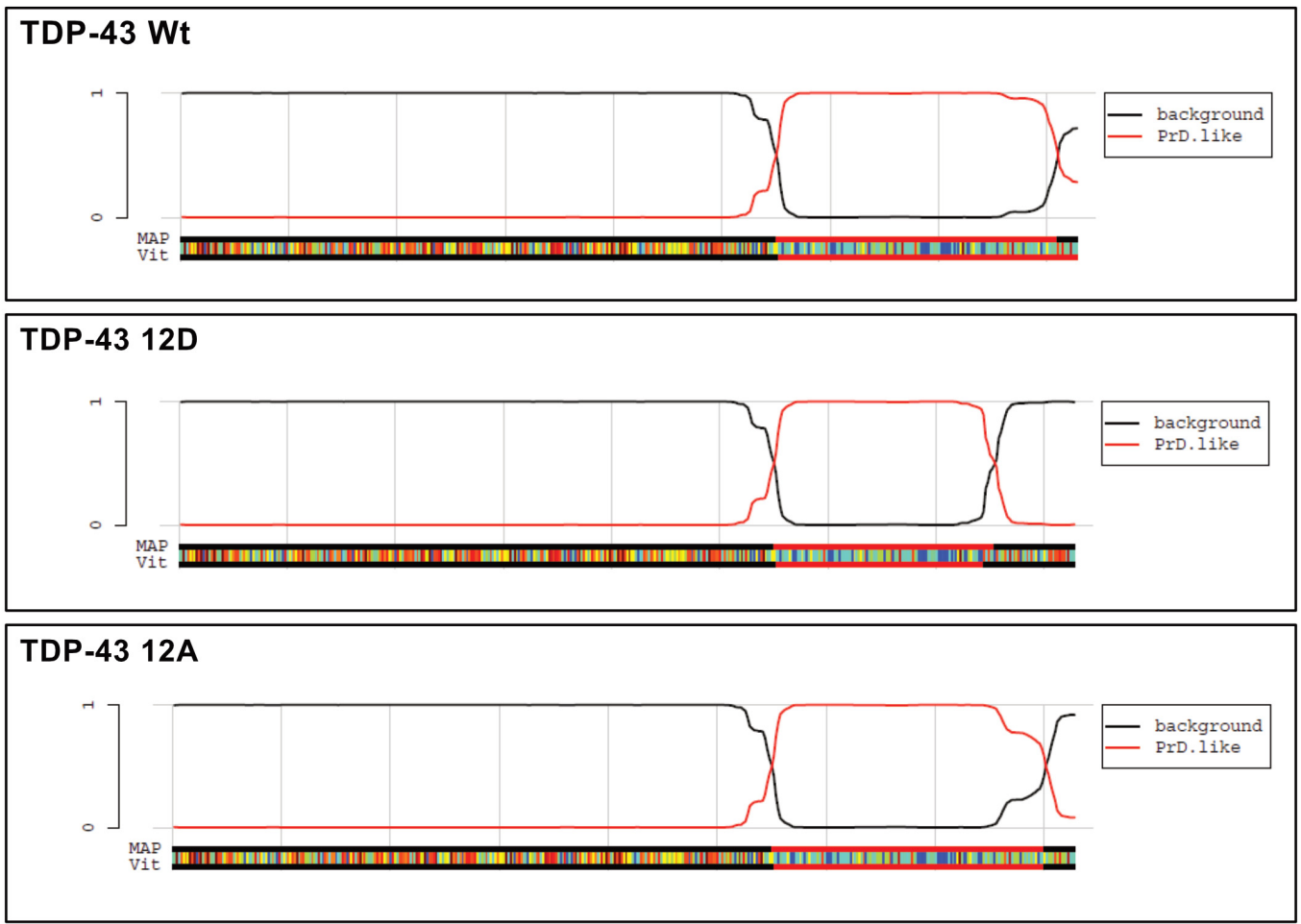

N Q Y G M S P A H T F R V I L K C W E

Fig. S3. PLAAC predicts different prion sub-sequences on TDP-43 Wt vs. phosphomimetic TDP-43 12D.

Visualization outputs from PLAAC, a web application that scans protein sequences for domains with prion-like amino acid composition (Lancaster et al., 2014). Each box shows a detailed visualization of the TDP-43 protein variants (TDP-43 Wt, 12D and 12A) and respective Hidden Markov Model (HMM) prion-prediction score, showing that the prion-like character of the TDP-43 LCD is reduced by the 12 phosphomimetic S-to-D mutations. On the bottom, each amino acid is color-coded by its enrichment log-likelihood ratio in PrLDs with HMM parse indicated by outer bars (black and red). 

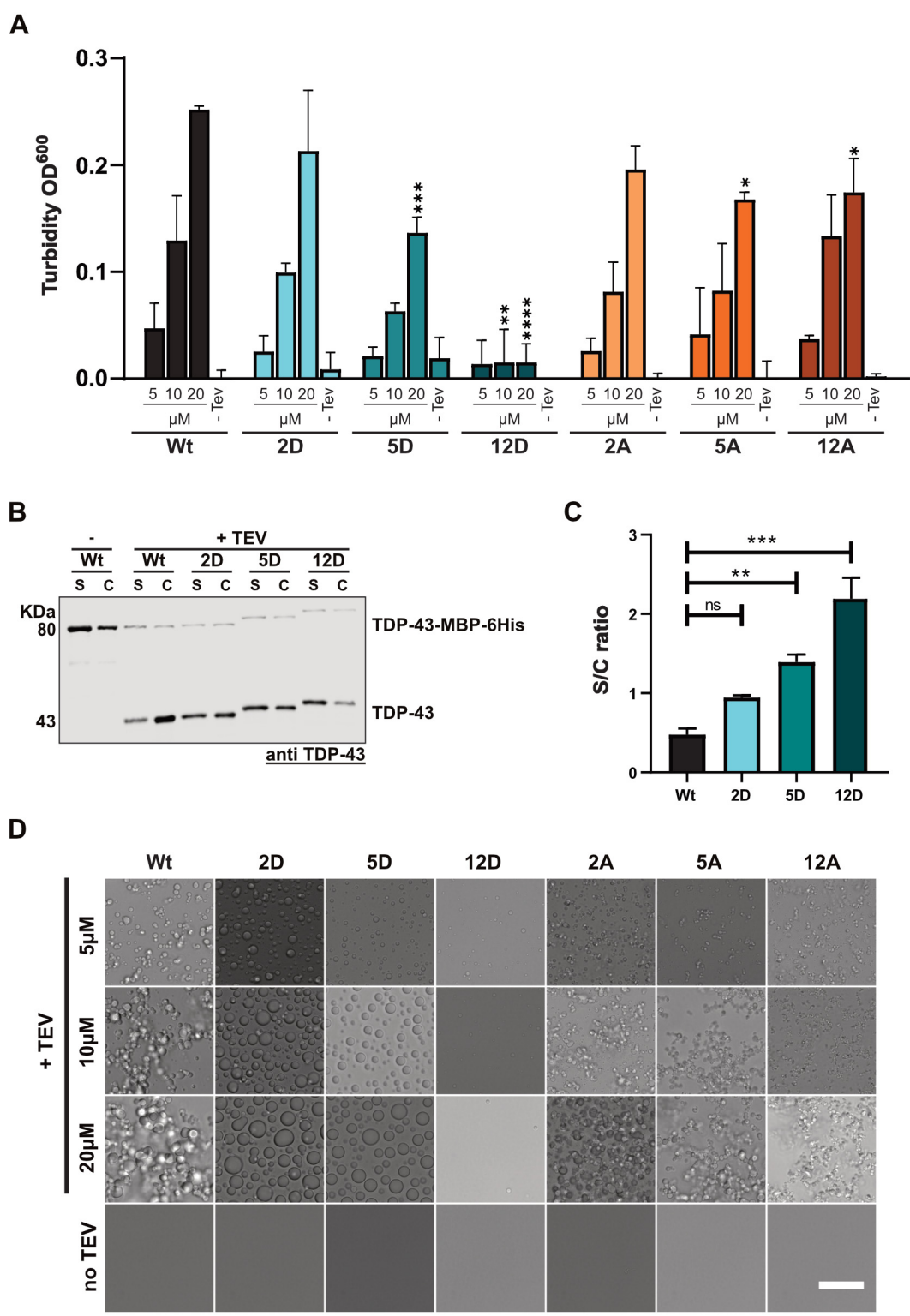

Fig. S4. C-terminal phosphomimetic substitutions reduce TDP-43 condensation in vitro.

A Turbidity measurements to quantify phase separation of different S-to-D and S-to-A mutants in comparison to TDP-43 Wt using phosphate buffer. Values represent means $(n=3) \pm$ SD. ${ }^{*} p$ $<0.0332, * * \mathrm{p}<0.0021, * * * \mathrm{p}<0.0002$ and $* * * * \mathrm{p}<0.0001$ by one-way ANOVA with Dunnett's multiple comparison test to Wt.

B Sedimentation assay to quantify condensation of S-to-D mutants in comparison to TDP-43 Wt (in Hepes buffer). TDP-43 was detected by TDP-43 Western blot (rabbit anti-TDP-43 Nterm).

C Quantification of band intensities corresponding to supernatant (S) and condensates (C) fractions is shown as means of $\mathrm{S} / \mathrm{C}$ ratio $(\mathrm{n}=3) \pm$ SEM. $* * \mathrm{p}<0.0021$ and $* * * \mathrm{p}<0.0002$ by one-way ANOVA with Dunnett's multiple comparison test to Wt.

D Representative bright field microscopic images of TDP-43 condensates in phosphate buffer (Bar, $25 \mu \mathrm{m}$ ). 


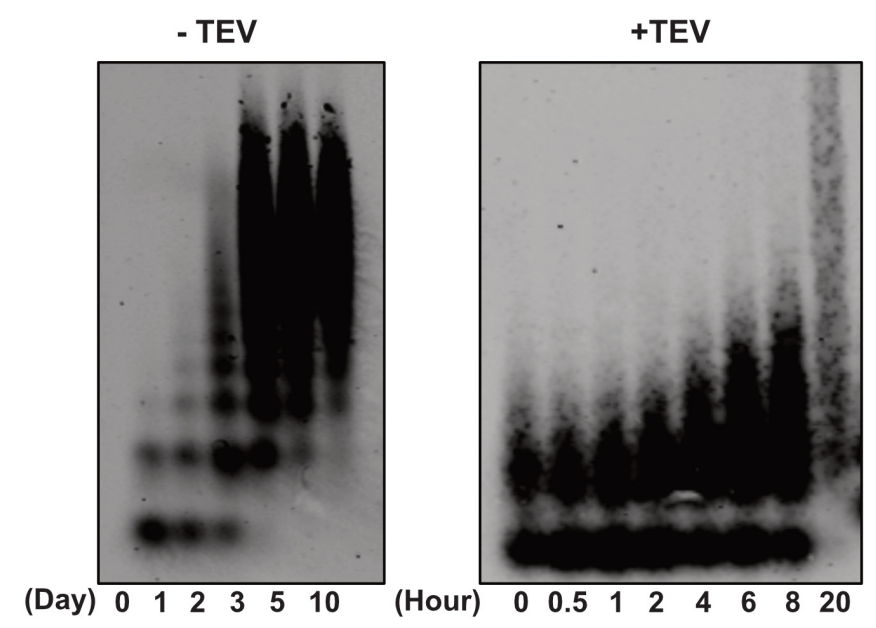

Fig. S5. Comparison of SDD-AGE analysis of TDP-43-MBP-His 6 with and without TEV protease-mediated cleavage.

In vitro aggregation assay of TDP-43-MBP-His 6 in the absence of TEV protease (-TEV) reveals the appearance of different SDS-resistant oligomers / high molecular weight species at late time points (at least 3 days of incubation). In comparison, when TDP-43-MBP-His6 is cleaved with TEV protease (+TEV), SDS-resistant oligomers / high molecular weight species already can be detected after several hours. Detection was performed after SDD-AGE analysis by anti-TDP43 Western blot (rabbit anti-TDP-43 N-term). 
A

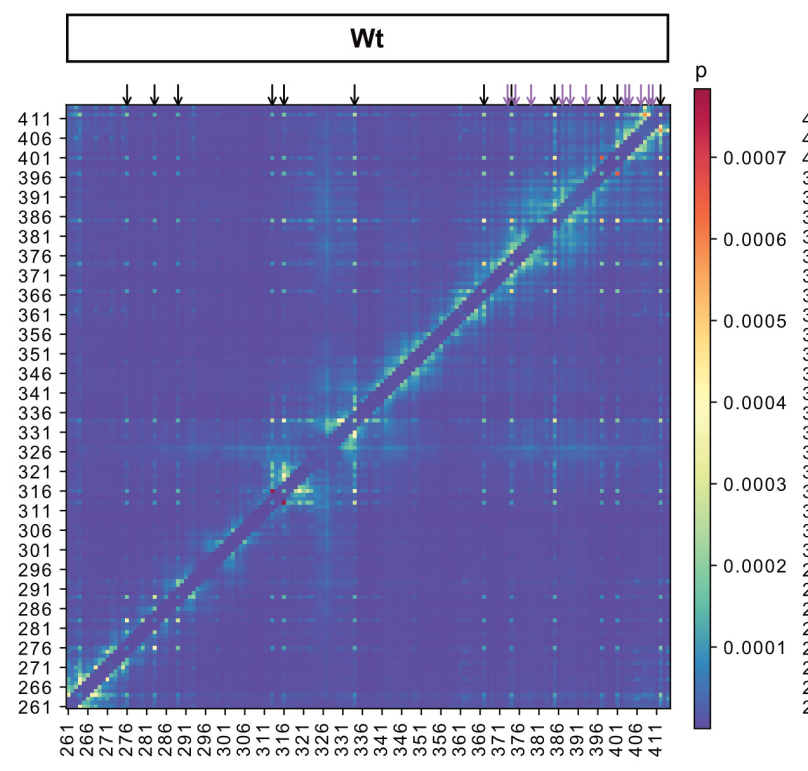

B

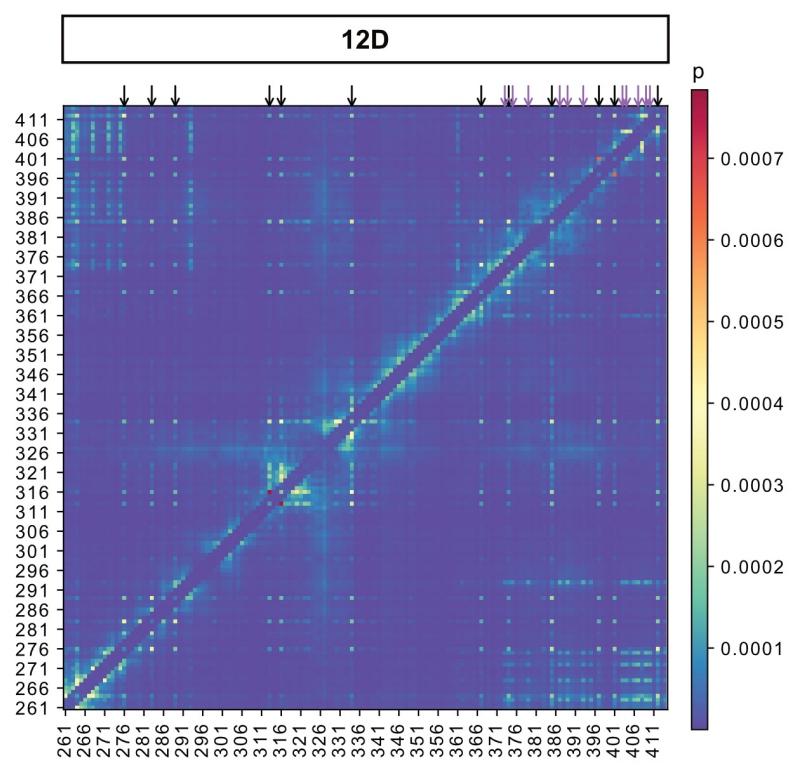

C

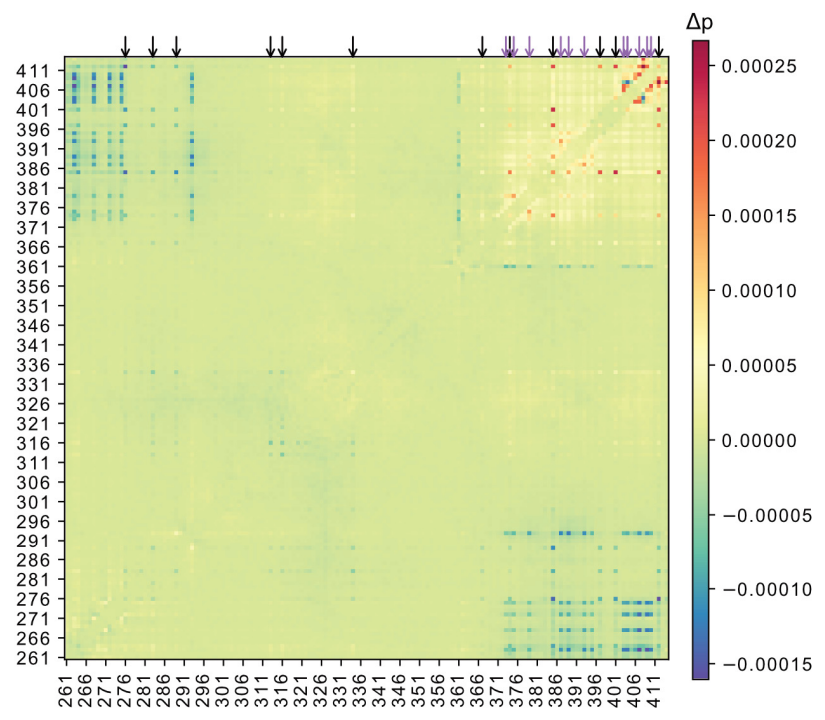

Fig. S6. Analysis of contacts in biomolecular condensates formed by the TDP-43 LCD in coarse-grained simulations.

A, B Contact maps for Wt (A) and 12D (B) TDP-43 LCD. Residue $\mathrm{i}$ and residue $\mathrm{j}$ are defined to be in contact if any of the coarse-grained beads are within $4.5 \AA$. The relative contact probability is calculated by averaging over all 118 protein chains and the last $5 \mu \mathrm{s}$ of $20 \mu \mathrm{s}$ simulations each. Intra-chain contacts with the two preceding and following residues are excluded from the analysis. Aromatic residues form prominent contacts and are highlighted by black arrows. E.g., looking at the column for F276 and following it upwards one can see that F276 interacts with F276 in other chains and irrespective of the chain, with F283, F289, F313, F316, W334, F367, Y347, W385, F401, and W412. The sites of the phosphomicking S-to-D mutations are highlighted by purple arrows. At these sites differences between Wt and 12D LCD can be seen, with Wt forming more contacts close in protein sequence and 12D instead interacting with R268, R272, R275, R293, and R361 further away in the sequence. 
bioRxiv preprint doi: https://doi.org/10.1101/2021.04.30.442163; this version posted April 30, 2021. The copyright holder for this preprint (which was not certified by peer review) is the author/funder. All rights reserved. No reuse allowed without permission.

C Differences in contact probability delta $p \_i, j=p \_i, j(W t)-p \_i, j(12 D)$ highlight that wild-type $\mathrm{S}$ residues, unlike phosphomicking $\mathrm{D}$ residues, favor interactions with residues close in sequence, while demonstrating that most contacts are not affected by the phosphomicking Sto-D mutations. 
A

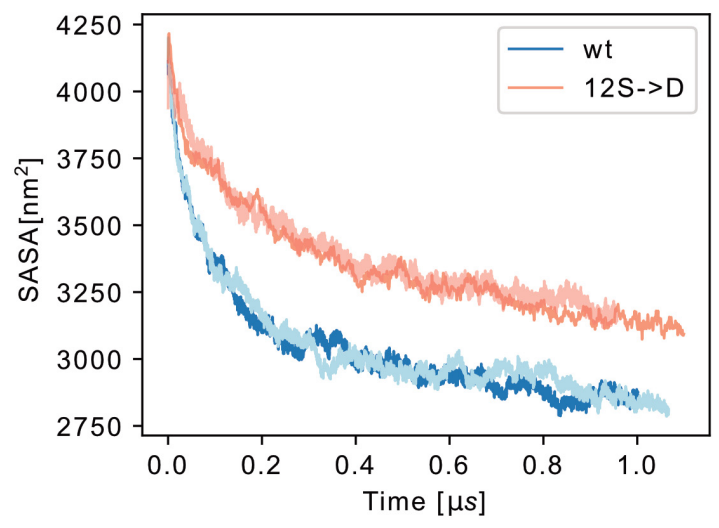

B
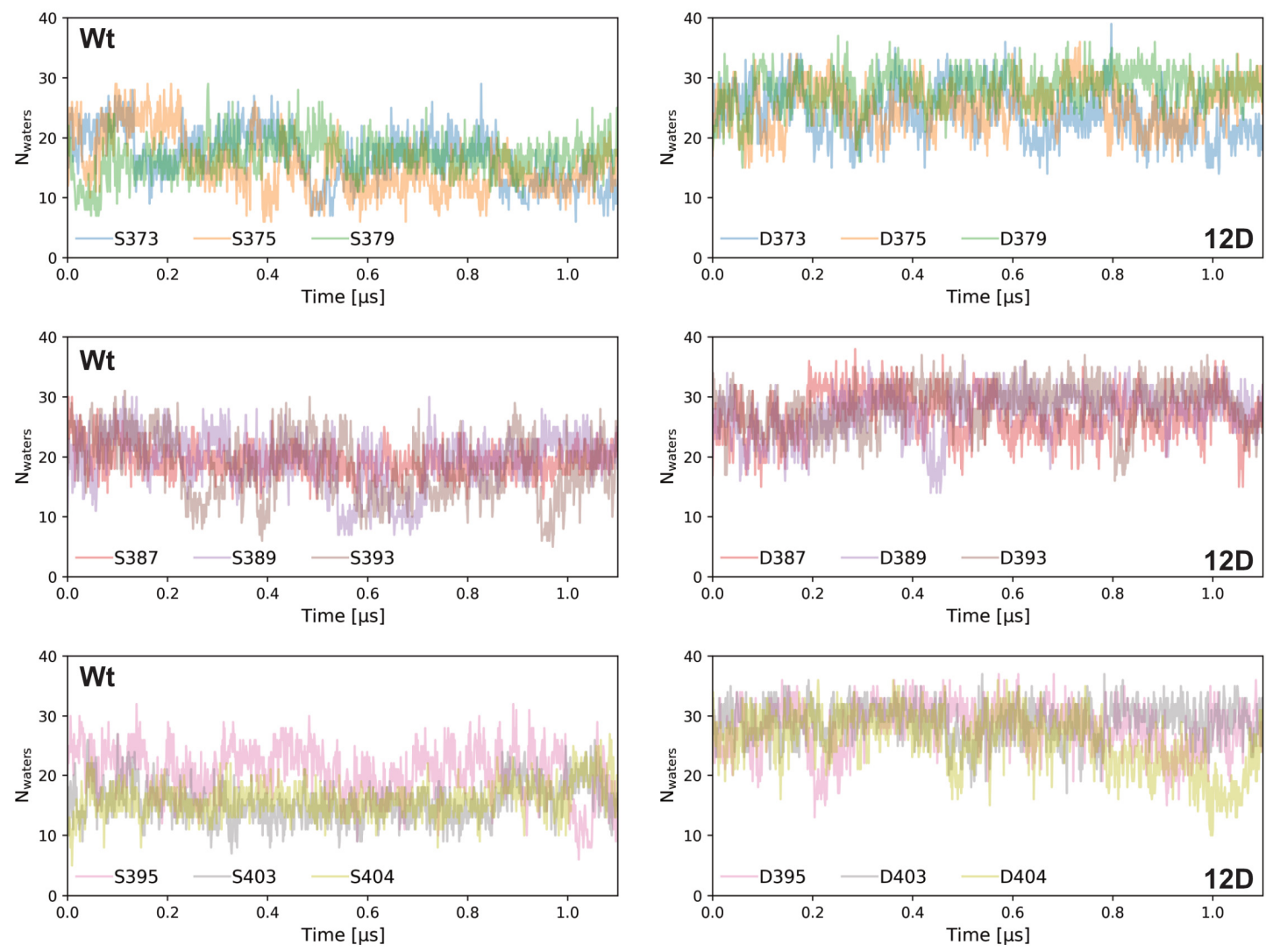

Fig. S7. Differences in protein-protein and protein-water interactions in atomistic simulations of dense clusters of Wt and 12D TDP-43 LCDs.

A Solvent-accessible surface area (SASA) of the LCDs of Wt (blue and light blue) and 12D mutant (red and salmon) in the simulations of their dense clusters as in biomolecular condensates. 12D mutant LCDs are more solvent-exposed than Wt LCDs, which is in line with the Wt LCDs forming stronger homotypic interactions.

B Dynamics of sidechain-water interactions at the sites of phosphomimicking $\mathrm{S}->\mathrm{D}$ substitutions in atomistic simulations of Wt and 12D LCD clusters. Numbers of waters bound to sites of phosphomimicking S-to-D mutations (within $5 \AA$ of the sidechain) are tracked over time for Wt (left) and 12D LCDs (right). While interactions are dynamic, there is a consistent trend with the phosphomimicking Asp residues binding more water molecules than the Ser residues in Wt LCDs. 
A

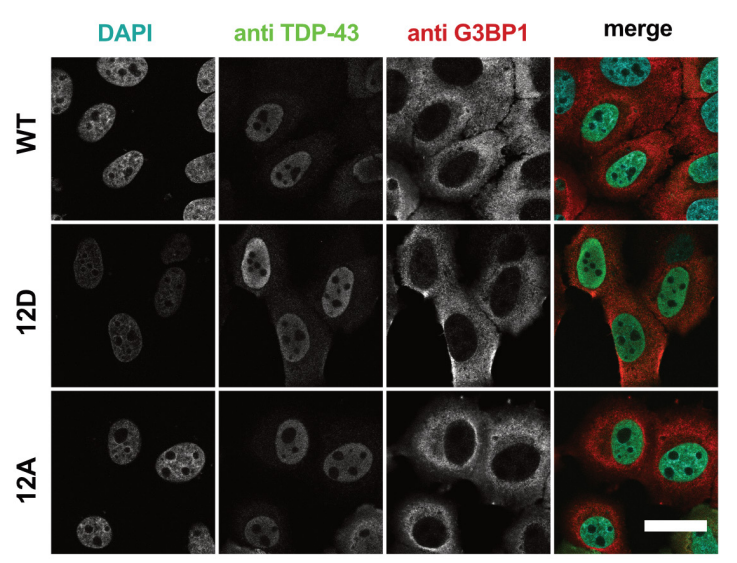

C

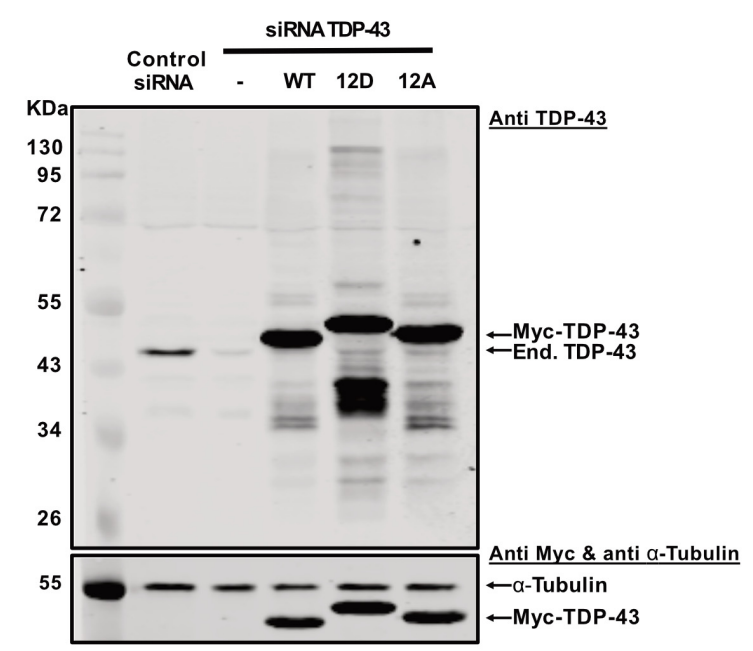

B

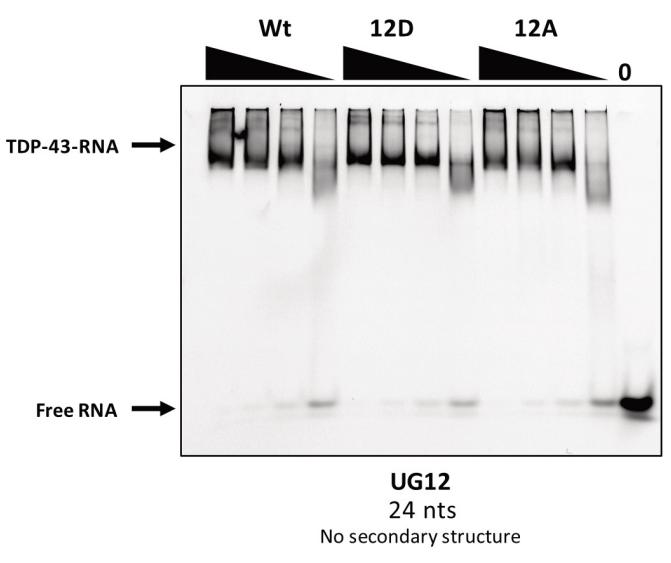

Fig. S8. Phosphomimetic substitutions do not alter nuclear localization and UG-rich RNA binding of TDP-43. Control experiment for siRNA-mediated TDP-43 knockdown and reexpression of myc-tagged TDP-43 variants.

A Immunostainings showing nuclear localization of TDP-43 Wt, 12D and 12A in HeLa cells. Endogenous TDP-43 expression was silenced by siRNAs, followed by transient transfection of the indicated siRNA-resistant myc-TDP-43 constructs. After $24 \mathrm{~h}$, localization of TDP-43 Wt, $12 \mathrm{D}$ and $12 \mathrm{~A}$ variants was visualized by TDP-43 immunostaining (mouse anti-TDP-43 antibody, Proteintech). G3BP1 (rabbit anti-G3BP1 antibody, Proteintech) and DAPI signal is shown to visualize the cytoplasm and nuclei, respectively. In the merge (right column), DAPI is show in turquoise, TDP-43 in green, and G3BP1 in magenta. Bar, $30 \mu \mathrm{m}$.

B Electrophoretic mobility shift assay (EMSA) of TDP-43-MBP-His6 variants (Wt, 12D and $12 \mathrm{~A})$ in a complex with (UG) 12 RNA.

C SDS-PAGE followed by TDP-43 Western blot (upper blot) showing efficient siRNAmediated knockdown of endogenous TDP-43 (running at $\sim 43 \mathrm{kDa}$ ) in comparison to control siRNA and re-expression of myc-tagged TDP-43 Wt, 12D and 12A in Hela cells. Equal loading is demonstrated by $\alpha$-Tubulin Western blot (bottom). TDP-43 was detected using rabbit antiTDP-43 C-term antibody (Proteintech), $\alpha$-Tubulin using mouse anti-alpha Tubulin antibody (Proteintech) and Myc-tag using mouse-anti Myc antibody (9E10, Helmholtz Center Munich). 
A

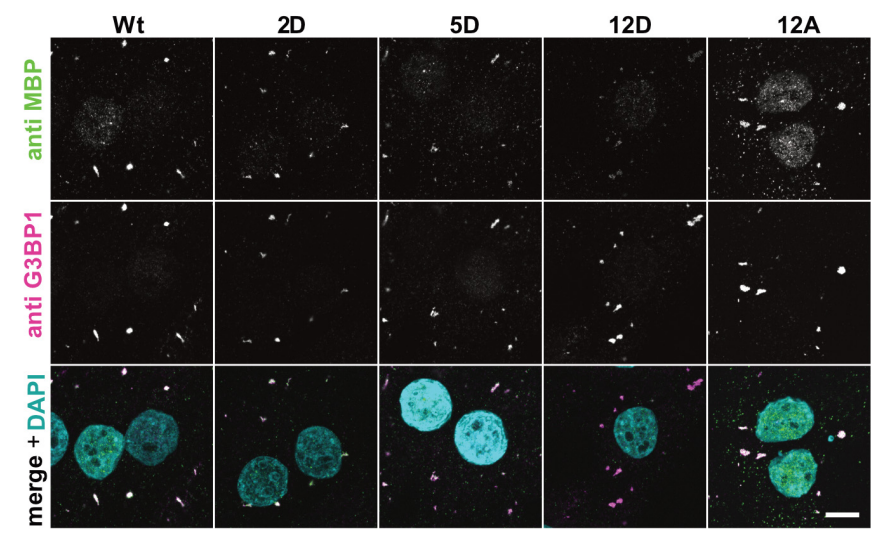

C

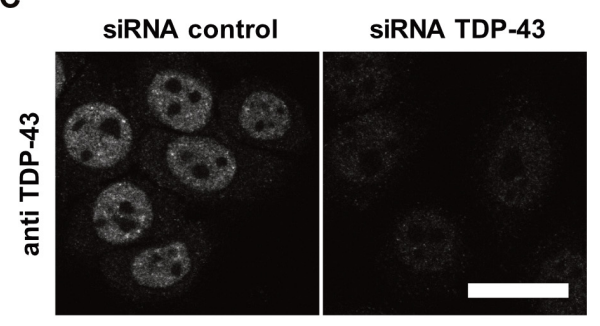

D
B
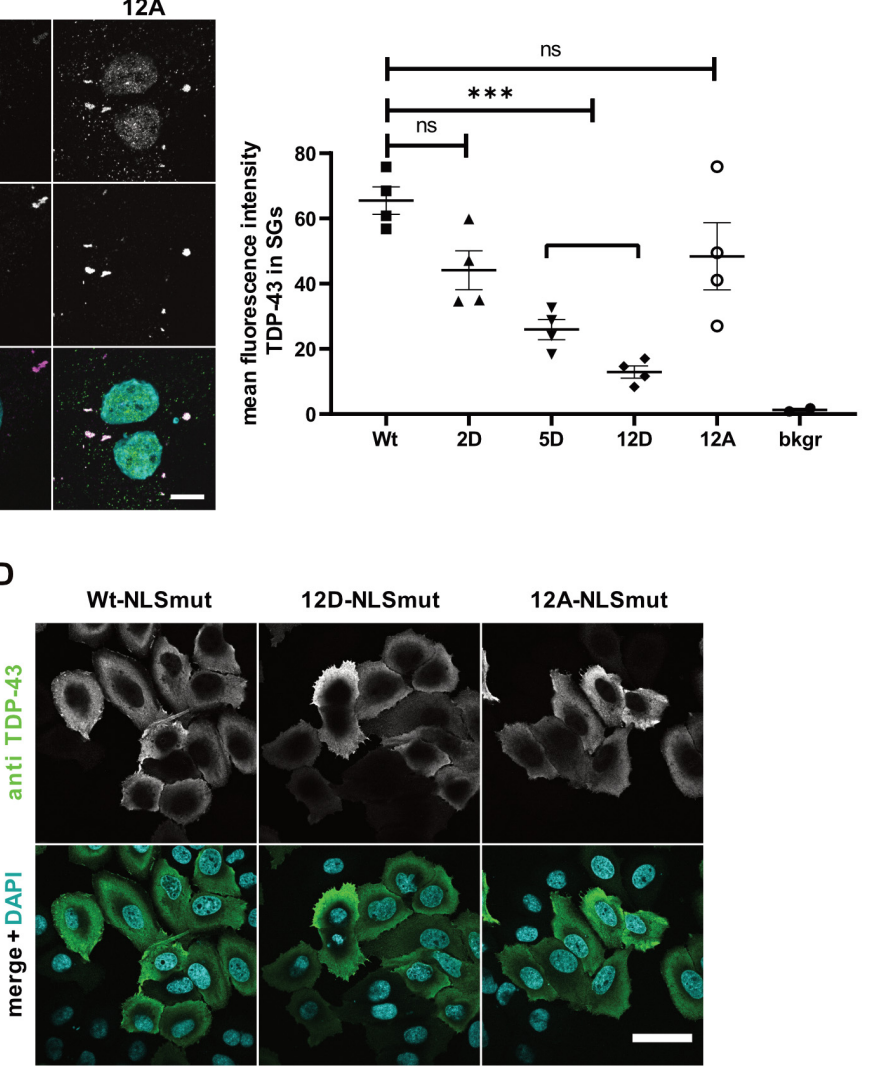

Fig. S9. Phosphomimetic S-to-D substitutions reduce association of TDP-43 with stress granules in semi-permeabilized cells. Control experiments for siRNA-mediated knockdown and localization of TDP-43-NLS mutants.

A Association of TDP-43 with stress granules (SGs) in semi-permeabilized HeLa cells is suppressed by phosphomimetic (2D, 5D and 12D) mutations in comparison to TDP-43 Wt and 12A. SGs and TDP-43-MBP-His6 were visualized by G3BP1 and MBP immunostaining, respectively. For clarity, signals were converted to grey values in the individual channels (upper two rows). In the merge (lower row), G3BP1 is shown in magenta, TDP-43-MBP-His 6 in green, white pixels indicate colocalization. Nuclei were counterstained with DAPI (turquoise). Bar, $10 \mu \mathrm{m}$.

B Quantification of the mean fluorescence intensity of TDP-43-MBP-His6 in SGs normalized to $\mathrm{Wt}$ for three independent replicates $\pm \mathrm{SEM}, * * * \mathrm{p}<0.0002$ defined by 1 -way ANOVA with Dunnett's multiple comparison test ( $\geq 10$ cells; $\geq 46$ SGs per condition).

C Representative confocal images showing efficient siRNA-mediated knockdown of endogenous TDP-43 versus control siRNA transfection. Endogenous TDP-43 (grey) was detected using mouse anti-TDP-43 antibody (Proteintech). Bar, $25 \mu \mathrm{m}$.

D Confocal images demonstrating equal cytoplasmic localization of TDP-43-NLS mutants (NLSmut Wt, 12D and 12A) $24 \mathrm{~h}$ after transfection and $48 \mathrm{~h}$ after endogenous TDP-43 silencing. Staining was carried out with mouse anti-TDP-43 antibody (Proteintech), signal shown in grey (upper row) or green (lower row); DAPI shown in turquoise. Bar, $40 \mu \mathrm{m}$. 


\section{Movie 1.}

Fluorescently labelled TDP-43 Wt condensates imaged live by spinning disc confocal microcopy.

Movie 2.

Fluorescently labelled TDP-43 5D condensates imaged live by spinning disc confocal microcopy.

\section{Movie 3.}

Fluorescently labelled TDP-43 12D condensates imaged live by spinning disc confocal microcopy.

\section{Movie 4.}

Coarse-grained simulations of TDP-43 LCD Wt with explicit solvent.

\section{Movie 5.}

All-atom simulations of TDP-43 LCD 12D with explicit representation of proteins, ions, and water with atomic resolution. 